\title{
Multiplicity in transiting planet-host stars
}

\section{A lucky imaging study of Kepler candidates}

\author{
J. Lillo-Box ${ }^{1}$, D. Barrado ${ }^{1,2}$, and H. Bouy ${ }^{1}$ \\ 1 Departamento de Astrofísica, Centro de Astrobiología, ESAC campus 28691 Villanueva de la Cañada, Madrid, Spain \\ e-mail: Jorge.Lillo@cab.inta-csic.es \\ 2 Centro Astronómico Hispano-Alemán (CAHA). Calar Alto Observatory, c/ Jesús Durbán Remón 2-2, 04004 Almería, Spain
}

Received 19 May 2012 / Accepted 1 August 2012

\section{ABSTRACT}

\begin{abstract}
Context. In the exoplanetary era, the Kepler spacecraft is causing a revolution by discovering thousands of new planet candidates. However, a follow-up program is needed to reject false candidates and fully characterize the bona-fide exoplanets.

Aims. Our main aims are to 1./ detect and analyze close companions inside the typical Kepler point spread function (PSF) to study whether they are the responsible for the dimming found in Kepler light curves, 2./ study the change in the stellar and planetary parameters caused by an unresolved object, 3./ help validate the Kepler objects of interest (KOI) that do not have any object inside the Kepler PSF, and 4./ study the multiplicity rate of planet-host candidates. Such a large sample of observed planet-host candidates allows us to derive statistics for close (visual or bounded) companions to the harboring star.

Methods. We present lucky imaging observations for a total of 98 KOIs. This technique is based on the acquisition of thousands of very-short-exposure-time images. A selection and combination of a small amount of the highest quality frames provides a high resolution image with objects having a $0.1 \operatorname{arcsec}$ PSF. We apply this technique to carry out observations in the Sloan $i$ and $z$ filters of our Kepler candidates.

Results. We find blended objects inside the Kepler PSF for a significant percentage of KOIs. On the one hand, only $58.2 \%$ of the hosts do not have any object within 6 arcsec. On the other hand, we find 19 companions closer than 3 arcsec in 17 KOIs. According to their magnitudes and $i-z$ colors, 8 of them could be physically bound to the host star.
\end{abstract}

Key words. binaries: visual - instrumentation: high angular resolution - planets and satellites: fundamental parameters

\section{Introduction}

It is not so long since the first discoveries of extrasolar planets. However, after the first detection of an exoplanet orbiting a main-sequence star (Mayor \& Queloz 1995; Marcy \& Butler 1996), the scientific community has discovered and confirmed hundreds of these objects around other stars. In this context, the Kepler Space Telescope has detected a large sample of new planet-host candidates by continuously observing more than 150000 stars in a specific field of view (RA $=19 \mathrm{~h} 22 \mathrm{~m} 40 \mathrm{~s}$ Dec $\left.=+44^{\circ} 30^{\prime} 00^{\prime \prime}\right)$. During the first five quarters of observations (i.e., $\approx 4.5$ months), the Kepler team collected in its second public release a total amount of 997 planet-host star candidates (second public release, Borucki et al. 2011).

However, to date, fewer than $5 \%$ of these candidates have been confirmed. After the candidate selection, there is a mandatory step to reject false positives before attempting any highly accurate (difficult and expensive) radial velocity measurements. Since the Kepler effective point-spread function (PSF) is very large (6-10 arcsec, depending on the particular target) and its pixel size is about 4 arcsec, several background objects could be blended with the host candidate (called Kepler object of interest, hereafter KOI). Bounded or not, these objects clearly affect the star and planet parameters such as the planet-to-star radius $\left(R_{\mathrm{p}} / R_{*}\right)$, semi-major axis $\left(a / R_{*}\right)$, impact parameter $(b)$, and even the planetary mass $\left(M_{\mathrm{p}}\right)$.

The presence of a secondary star could lead, for example, to the definite rejection of the candidate (see O'Donovan et al. 2006) There are several configurations that can mimic an exoplanet transit around its host star. The most relevant are A./ a small substellar object transiting the other component of the binary system, since the smallest stars and brown dwarfs have the same size as Jupiter; B./ a stellar binary blended with a background star; C./ a grazing binary, which has not been ruled out by additional photometry or spectroscopy; D./ a background eclipsing binary blended by the light of the KOI; E./ a larger planet transiting a background star blended by the main target (would not strictly be a false positive since there is a planet in the system but would be in the sense that it rejects the brightest central star as a planet-host); and F./ a long-term spot. There would be a "G-case" where the main target actually hosts a planet but with either a blended background companion or a nontransiting bounded companion. These configurations would lead to a change in the planet properties, as we said before.

Some of these configurations might be ruled out by the automatic pipeline implemented by the Kepler team (Jenkins et al. 2010). While cases A and B are clearly rejected by this pipeline and an individual study of the light curves done by the team itself (Borucki et al. 2011), low-resolution spectroscopy clearly reject the $\mathrm{C}$ configuration. However, the $\mathrm{D}, \mathrm{E}$, and $\mathrm{G}$ cases are the main sources of false positives in the sample of transiting planet candidates. More specifically, case $\mathrm{G}$ clearly shows the need for an intense high-resolution imaging follow-up program to validate the planetary nature of the transients. Owing to the Kepler long base-line, we expect few or no F cases.

Theoretical studies of the false positive probability of Kepler candidates conclude that obtaining high resolution images down to $1-2$ arcsec is crucial for confirming the planetary detections 
and their physical properties. As an example, an Earth-size planet transiting a faint star might have a false positive probability greater that $20 \%$ if it lacks high resolution imaging, which could potentially be decreased to less than $2 \%$ with a high resolution image (Morton \& Johnson 2011). Several authors have acquired this kind of observation for other planet-host candidates finding significantly different planet-star properties. For instance, Daemgen et al. (2009) found stellar companions to three stars harboring planets. As a consequence, the updated values of the physical parameters differ by about $2 \%$ from the previous ones.

However, even if the planet is confirmed, its formation and evolution scenarios (including its migration) require an accurate description of the effect of bounded stellar companions. The vast majority of planets found in multiple systems are actually S-type (the planet orbits one of the two components of the system, and the other component plays the role of a gravitational perturber, see Kley 2010). These secondary objects make planet formation difficult since they interact dynamically with the system elements producing an extra heating of the protoplanetary disk. All of these factors may cause large changes in the planetary architecture and exoplanetary properties from those present when the planets formed around single stars. For instance, Eggenberger et al. (2004) found a statistical segregation in the planet mass for planets with orbital periods shorter than 40 days around single and multiple systems.

In this paper, we present the first statistical study of multiplicity in Kepler exoplanetary candidates. A set of high resolution images obtained with the lucky imaging technique (Law et al. 2006) at the $2.2 \mathrm{~m}$ telescope in Calar Alto Observatory (Almería, Spain) with the AstraLux instrument was acquired. This technique allows us to obtain diffraction-limited observations with the best seeing conditions in the Sloan Digital Sky Survey (SDSS) $i$ band (see Félix Hormuth's Ph.D. dissertation). A total of 98 KOIs (i.e., about $10 \%$ of the candidates listed in the second public release of the Kepler team) have been targeted and studied.

In Sect. 2, we explain the observations, image processing, and data extraction from the raw images, in terms of sample selection (Sect. 2.1), data acquisition (Sect. 2.2), data reduction, and photometric calibration (Sect. 2.3), including the astrometric corrections, and a study of the sensitivity curves and detectability of our observations. The results based on these observations are presented in Sect. 3. We also perform spectral typing of the detected companions (Sect. 4.1) and investigate the possible gravitational bound between some of them (Sect. 4.2). In Sect. 4.3, we discuss the implications of the calculated planetary parameters from the Kepler light curves for the possible presence of a blended star. Some particularly interesting cases are studied in Sect. 4.4 and the final conclusions of this work are presented in Sect. 5.

\section{Observations and data reduction}

\subsection{Sample selection}

We observed 98 KOIs. Owing to the lack of any kind of followup observations the majority of them were selected from the published data of the Kepler team (Borucki et al. 2011, second public release). The selection of the rest was coordinated with the Kepler team. In addition, we limited our targets to be brighter than $15 \mathrm{mag}$ in the Sloan $i$ band in order to detect objects 5 mag fainter than the KOI (see Sect. 2.3.6). Apart from these constraints, KOIs were randomly selected from the remaining sample so we expect no bias in our results.

\subsection{Data acquisition}

The data presented in this paper were taken at the Calar Alto Observatory (Almería, Spain) during 19 nights of observations divided into three separate runs (6-12 June 2011, 1-10 July 2011, and 25-26 July 2011). Although two nights were lost owing to adverse weather conditions, the remaining 17 had very good atmospheric stability with low atmospheric extinction and either sporadic or absent clouds, which are ideal for this observing technique. The mean natural seeing was around $0.9 \operatorname{arcsec}$ over the entire run.

We used the AstraLux instrument placed at the $2.2 \mathrm{~m}$ telescope to perform diffraction-limited imaging of our sample of KOIs with the lucky imaging technique. We used the entire available field of view of the camera (i.e., $24 \times 24$ arcsec) to cover a separation range encompassing the entire mean Kepler PSF (6-10 arcsec). In brief, we took thousands of images with short exposure times (100-200 ms), well below the typical timescale on which the atmospheric turbulence changes (see Felix Hormuth's Ph.D. dissertation). We set the exposure times and number of frames according to the atmospheric conditions and target magnitude in order to reach at least 5.0 mag fainter than the central KOI. Thus, our total exposure times were in the range 340-2000 s. We note that objects fainter than this magnitude difference would not affect the planet-star properties by more than $0.5 \%$ in the case of the $R_{\mathrm{p}} / R_{*}$ rate (see Eq. (6) in Seager \& Mallén-Ornelas 2003). Moreover, the probability that Kepler observations have detected a transit with a signal-to-noise ratio $(\mathrm{S} / \mathrm{N})$ greater than 7.1 decreases as the star gets fainter. Thus, we designed our observations to reach $\Delta m_{i} \approx 5.0 \mathrm{mag}$ $1.0-1.5$ arcsec from the KOI.

Images were firstly obtained in the SDSS $i$ filter since the PSF deformation is lower for this band than for shorter wavelengths (this result is quite similar for the SDSSz band). An on-the-fly reduction allowed us to immediately check for close sources. If any were present, $\mathrm{SDSS} z$ band images were acquired in order to characterize the secondary object by using its $i-z$ color (see Sect. 2.3.5).

\subsection{Data reduction and photometric calibration}

\subsubsection{Basic reduction}

We used the AstraLux pipeline (see Hormuth 2007) ${ }^{1}$ to perform the basic reduction and combination of our lucky imaging frames. First, this pipeline applies the bias and flat-field correction corresponding to each night of the science images. We used the dome flat-field to avoid inhomogeneities due to the passing of some clouds during sunset for most of the observing nights. Second, it measures the quality of each science frame to select the $1.0 \%, 2.5 \%, 5.0 \%$, and $10 \%$ frames with the highest Strehl ratios (Strehl 1902), calculates the shifts between these single frames, performs the stacking, and divides the pixel size in half (i.e., the pixel scale is resampled from $0.0466^{\prime \prime} /$ pixel to $0.0233^{\prime \prime} /$ pixel). According to our observing configuration (number of frames, single exposure time, and gain), we need to use the $10 \%$ selection-rate images to reduce the photometric errors (as recommended in the user's manual). Inspection of the lower

\footnotetext{
http://www.mpia-hd.mpg.de/ASTRALUX/
} 
Table 1. Plate solution for our photometric observations.

\begin{tabular}{lcc}
\hline \hline Coeff. & $\xi$ & $\eta$ \\
\hline$c_{10}$ & $-23.58 \mathrm{mas} / \mathrm{px}$ & $-0.65 \mathrm{mas} / \mathrm{px}$ \\
$c_{01}$ & $-0.81 \mathrm{mas} / \mathrm{px}$ & $23.58 \mathrm{mas} / \mathrm{px}$ \\
$c_{20}$ & $4.05 \times 10^{-3} \mathrm{mas} / \mathrm{px}^{2}$ & $7.93 \times 10^{-5} \mathrm{mas} / \mathrm{px}^{2}$ \\
$c_{02}$ & $1.31 \times 10^{-3} \mathrm{mas} / \mathrm{px}^{2}$ & $9.35 \times 10^{-5} \mathrm{mas} / \mathrm{px}^{2}$ \\
\hline rms & $48.5 \mathrm{mas}$ & $34.5 \mathrm{mas}$ \\
PA & $1.78^{\circ}$ & \\
Pixel scale & $23.59 \mathrm{mas} / \mathrm{px}$ & \\
\hline
\end{tabular}

selection-rate images was done for source detection purposes but not for the photometric analysis presented in this paper.

\subsubsection{Astrometric corrections}

Astrometric corrections were applied to the positions of the targets when calculating angular separations and angles between them. We made use of our observations of the M 15 dense globular cluster. A total of 66 stars in our image were identified and matched with the Yanny et al. (1994) catalog from images of the Hubble Space Telescope. We performed an astrometric calibration using the ccmap package in IRAF. We computed the transformation between $[x, y]$ coordinates to the $[\xi, \eta]$ tangent plane coordinates in a second order fitting

$$
\begin{aligned}
& \xi=c_{00}+c_{10} x+c_{01} y+c_{20} x^{2}+c_{02} y^{2} \\
& \eta=c_{00}^{\prime}+c_{10}^{\prime} x+c_{01}^{\prime} y+c_{20}^{\prime} x^{2}+c_{02}^{\prime} y^{2}
\end{aligned}
$$

where $c_{i j}$ are the corresponding calculated coefficients, which are shown in Table 1. We found residuals of 48.5 mas in the $\xi$ direction and 34.5 mas in the $\eta$ direction. The pixel scale and rotation angle were found to be $0.02359 \pm 0.0005 \mathrm{arcsec} / \mathrm{pixel}$ and $1.78 \pm 0.01$ degrees, respectively. We considered the rms value as our mean astrometric error in both tangential coordinates.

\subsubsection{Source detection and photometric extraction}

The SExtractor software for source extraction (Bertin \& Arnouts 1996) was used to extract the pseudo-instrumental photometry (explained below) of sources surrounding each KOI. We chose 60 pixel apertures ( 1.4 arcsec) to account for the maximum flux percentage of the objects. We set the detection threshold to 2.0- $\sigma$ in a minimum area of 30 pixels. Since this software does not take into account the exposure time and electromagnetic gain of the observations, we had to correct the SExtractor output to obtain the real instrumental magnitudes. The gain correction was necessary since different gains were set to different objects to avoid detector saturation. This was the case for standard stars for which we set the software gain to be within 230-255. For all the science images, we applied a software gain of 255 , which we note, however, is neither equal nor linearly correlated to the physical electron gain (see Fig. 3.7 of Hormuth 2007). In a private communication, the PI of the instrument (Felix Hormuth) provided us with a calibrated function relating both parameters. Hence, referring to the physical electron gain as $Y$, the instrumental magnitude of an object would be: $m_{\text {inst }}=m_{\text {SExtractor }}+2.5 \log \left(Y t_{\text {exp }}\right)$.

\subsubsection{Atmospheric extinction and zero point calculation}

The extinction correction and determination of the zero points were done by using observations of SDSS standard stars (Smith et al. 2002). We observed Ross 711 and SA105-815 during the first run; Ross 711, SA105-815 and BD+25-4655 in the second run, and Ross 711 and $\mathrm{BD}+25-4655$ in the third run. A good sampling of the airmass for each night ensured a correct determination of the calibration parameters for the photometric nights, namely the extinction coefficient $(\chi)$ and the zero point $(C)$. According to the Calar Alto weather monitoring webpage $^{2}$ and the results of the calibration diagrams $\left(m_{\text {standard }}-m_{\text {inst }}\right.$ vs. airmass) for each night, we conclude that the following nights of the year 2011 were clearly photometric: July 5, 6, 7, 10, and 26. The average error in the calculated magnitudes for these nights is around $1 \%$. Another set of nights were classified as partially photometric because of sporadic clouds occurring during the observing time or irregular seeing conditions that led to inaccurate photometry, since we observed the standard stars frequently. These nights were June 11 and 12, and July 1, 3, and 9. Consequently, errors in these partially photometric nights are greater (1-3\%) but still acceptable for our purposes. The column \#5 in Table 5 provide information about the stability of the atmosphere for the observations of each object (according to the acquisition date). From our photometric results, we can conclude that magnitudes brighter than $i=21-22$ have neglegible errors while fainter objects are too noisy to accurately determine their magnitudes with errors below $1.5 \%$ and were then not taken into account for this study.

Hence, the calibrated magnitude for these objects was then calculated to be:

$m_{\mathrm{cal}}=m_{\mathrm{inst}}+2.5 \log \left(A_{\mathrm{p}} Y\right)+Z_{\mathrm{p}}+C \chi$,

where $A_{\mathrm{p}}$ is the aperture correction, $Y$ the electron gain, $C$ the extinction coefficient, $Z_{\mathrm{p}}$ the zero point, and $\chi$ is the correspondent airmass.

When compared to the Kepler Input Catalog (KIC) values provided in the Kepler MAST (Mikulski Archive for Space Telescopes $)^{3}$, we obtain magnitude differences $\left(i_{\mathrm{KIC}}-i_{\mathrm{AstraLux}}\right)$ smaller than $0.1 \mathrm{mag}$ for around $60 \%$ of our objects without close companions, where $90 \%$ of them are smaller than 0.4 mag.

\subsubsection{Detection and calibration of very close objects}

By closely examining the reduced images, we selected the subsample of our KOIs that had close companions within 3 arcsec (17 KOIs) to perform an individual extraction of the photometry. Owing to its proximity to the main target, we would have need to use smaller apertures in order to avoid contamination from the PSF wings of the KOI, hence, an aperture correction was applied. Contamination coming from the KOI's PSF wing was estimated by measuring the amount of flux from the KOI inside the companion's aperture. This extra flux influenced the magnitude of the companion in less than $0.03 \mathrm{mag}$, which is far smaller than the photometric errors of these sources. Hence, no corrections of this nature were performed in this work. Photometric extraction of the standard stars was also done with the IRAF package ${ }^{4}$ and calibration parameters were re-derived and applied to the science photometry. We determined the aperture correction by using the standard stars. As the phot task of the IRAF package already

\footnotetext{
Publicly available at www. caha.es/WDXI/wdxi.php

http://archive.stsci.edu/kepler/

4 Image Reduction and Analysis Facility, http://iraf.noao.edu/
} 


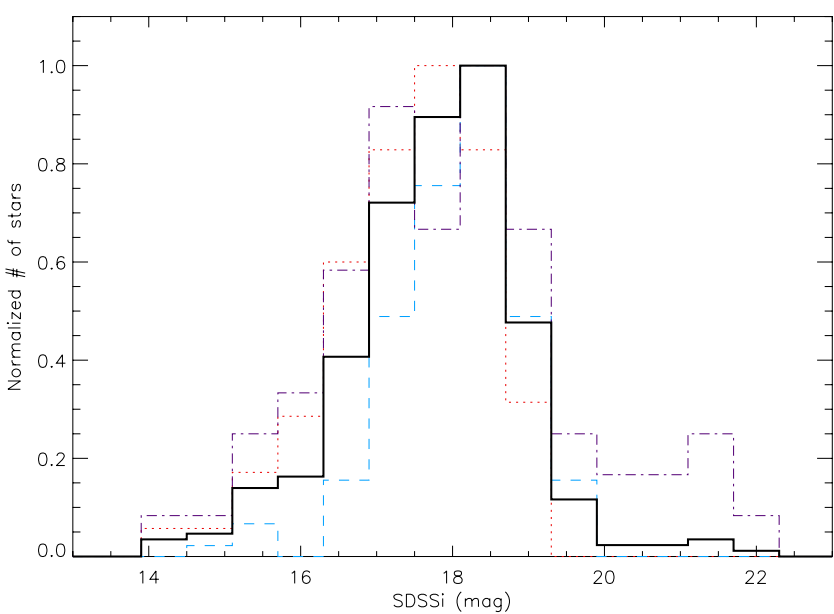

Fig. 1. Mean completeness and detectability limits of our survey (black solid line) for a $200 \mathrm{~s}$ effective exposure-time image. Red dotted line, blue dashed line, and purple dotted-dashed line represent the values for the three photometric nights considered, namely $26 \mathrm{Jul}$. 11, $05 \mathrm{Jul} .11$ and 07 Jul. 11, respectively. Bin size is $0.5 \mathrm{mag}$. The histograms have been normalized to their maximum value for visualization purposes.

takes into account the exposure time of the image, here we just had to correct for aperture correction, electron gain, and atmospheric extinction. Hence, the final expression for deriving the calibrated magnitudes is

$m_{\mathrm{cal}}=m_{\mathrm{IRAF}}+2.5 \log \left(A_{\mathrm{p}} Y\right)+Z_{\mathrm{p}}+C \chi$,

where $\chi$ is the corresponding airmass.

We also tested a PSF approach rather that aperture photometry. The main problem of this technique with lucky-imaging observations is to find a standard PSF for the whole night. Owing to the acquisition technique, each particular image/star would have a characteristic PSF that cannot be modeled by a general one. However, aperture photometry for very close $(<1.5$ arcsec $)$ and faint companions can involve larger errors even using small apertures. Hence, we decided to use PSF photometry for the 2 KOIs with faint companions at very small angular separations (KOI-1375 and KOI-0387). The PSF was measured for the different available standard stars during the whole night. We calculated the stellar fluxes for each PSF and estimated the relative magnitudes between the KOI and the companion ( $\Delta i$ and $\Delta z$ ) as a weighted average of all values. Since aperture and PSF photometry must coincide for the bright KOI, we used the calibrated photometry for the KOI obtained by aperture photometry $\left(m_{\mathrm{P}}\right)$ to obtain the companions' magnitudes as $m_{/ \mathrm{C}}=m_{\mathrm{P}}+\Delta m$ for each filter in these two cases. For the remaining objects, we used aperture photometry.

\subsubsection{Sensitivity, detectability, and limiting magnitudes}

We estimated the mean completeness and limiting magnitudes of our images by using the observations of the globular cluster M 15 in order to have a large sample of stars with a wide range of magnitudes. We measured the number of objects per magnitude bin of $0.5 \mathrm{mag}$ on every photometric night for which images of M15 were taken. Computing the mean values of these bins we were able to construct the histogram shown in Fig. 1. We scaled this histogram to a 200 -s exposure-time image to account for the mean exposure time of our science images. We obtained a mean completeness value of $i_{\text {complete }}=18.4 \pm 0.3 \mathrm{mag}$ and

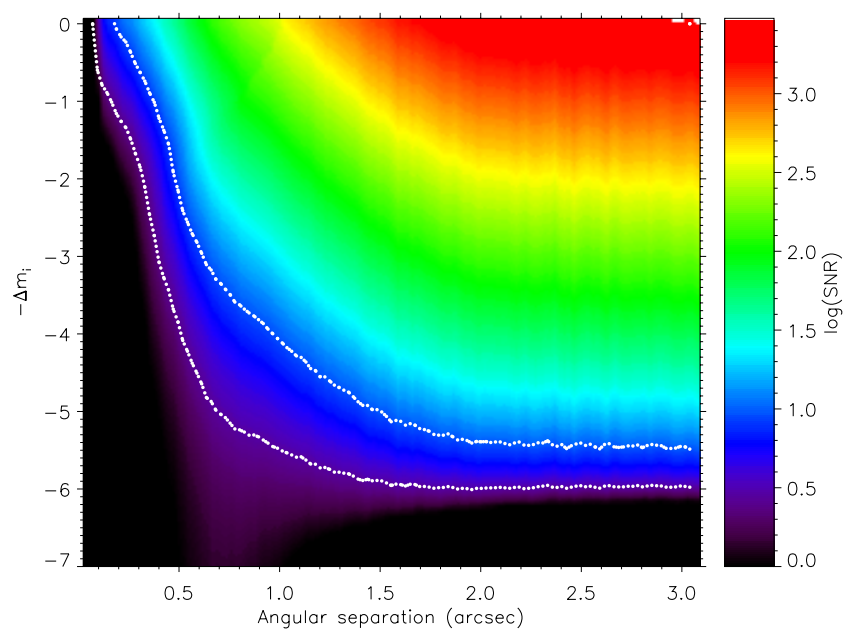

Fig. 2. An example of the sensitivity function for our AstraLux observations. We show the results for KOI-0717 for an image with the $10 \%$ selection rate. Color code represents the measured $\mathrm{S} / \mathrm{N}$ for the detection of the artificial companion profile. The white lines represent the contours for the 3- $\sigma$ (lower line) and 10- $\sigma$ (upper line) $\mathrm{S} / \mathrm{N}$ detections.

found that our faintest detected source has $i=22.5 \mathrm{mag}$. In the case of the SDSSz observations, we adapted the exposure time to detect the companion seen in the SDSSi image. It is important to remember that these values were calculated for a particular exposure time. Since we set different exposure times for each target in order to achieve $\Delta m_{i} \approx 5.0$ (see lower panel in Fig. 3), the completeness and detectability limits should be different for each image. For each particular science image,the scaled values of $i_{\text {complete }}$ related to its real exposure time are shown in Table 4. We note that we only show here the observations for the nonisolated KOIs (Kepler host candidates without any objects closer than 6 arcsec).

Sensitivity functions were compiled for each image to determine the depth in angular separation and magnitude (actually, $\Delta m$ ) to which we are complete for a 3- $\sigma$ detection threshold.

By measuring the observed radial profile averaged over a large number of directions (avoiding angles in the direction of close companions), we were able to infer the dependence of the primary star brightness along the angular separation. We then, reiteratively added the similar profiles but located at different angular separations in the range 0.2-3.0 arcsec and scaled them to be $\Delta m$ magnitudes fainter according to the expression $F_{/ \mathrm{C}}=F_{\mathrm{P}} 10^{-0.4 \Delta m}$, where $F_{/ \mathrm{C}}$ is the encircled flux of the companion and $F_{\mathrm{P}}$ the flux of the primary. We then measured the S/N of the detection of the added profile. Figure 2 shows an example of these calculations where the artificial companion to the observed KOI profile have differential magnitudes of $\Delta m=[0,7]$ and angular separations $0.2-3$ arcsec.

\section{Results}

We found 111 companion sources at angular separations of 0.3-10 arcsec from the 98 targeted KOIs. Since the Kepler PSF varies from 6 to 10 arcsec, we consider a KOI to be isolated if no objects smaller than 6 arcsec are found in our lucky imaging observations. Objects with larger angular separations could be easily detected by the Kepler images or previous ground-based all-sky surveys.

According to this definition, we detected 57 isolated KOIs. This means a $58.2 \%$ rate of isolated objects for planet-host 


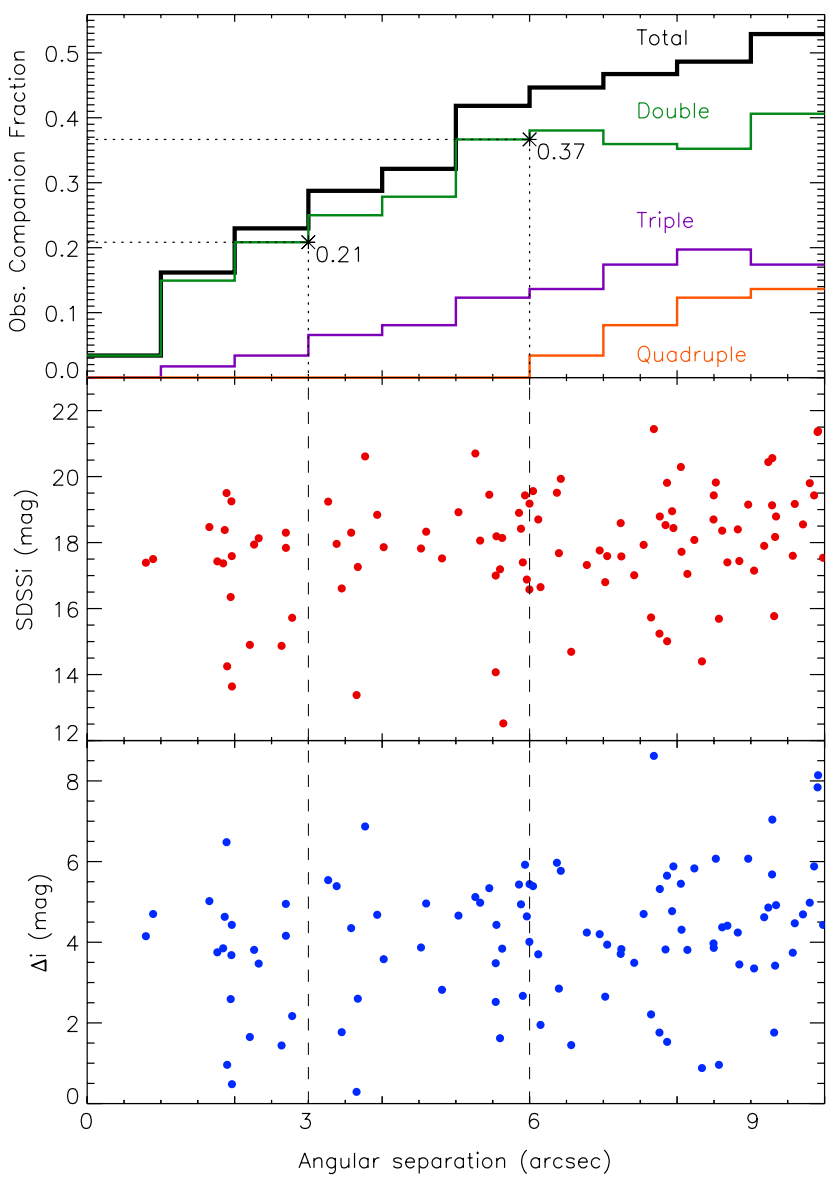

Fig. 3. Upper panel: cumulative observed-companion fraction (ocf, see text) for the double (green), triple (purple), and quadruple (orange) systems as well as the total ocf (black). We also show the values for the double system at 3 and 6 arcsec. Middle and lower panels: calibrated SDSSi magnitude of the 111 companions and differential magnitude with their KOIs. Dashed lines mark the 3 and 6 arcsec positions.

candidates in the Kepler objects of interest catalog. It is important to remark that this isolation rate would decrease to $33.7 \%$ if we considered a 10 arcsec PSF for Kepler observations. However, the most accurate PSF of 6 arcsec is considered in this paper.

Among the KOIs with visible companions at angular separations of 6 arcsec, 41 planet-host candidates have, at least, one object within this projected separation (i.e., 41.8\%). Among these, 17 KOIs have a total amount of 19 companions within 3 arcsec (17.3\% of all KOIs considered). We refer to this group in this paper as the close companions' group. In Fig. 5, we show the 17 KOIs with close companions and the Kepler optimal (public) apertures of the different quarters into which the Kepler mission is divided. All 19 companions lie within these apertures contaminating the light curve fluxes as well as the corresponding planetstar properties and planet validation. Moreover, 27 KOIs have 30 companions within the range $3-6$ arcsec $(27.6 \%$ of all KOIs considered), medium-distance group. This means that 3 of the main targets from the close companion group (KOI-0433, KOI0641, and KOI-0841, see Fig. 4) have either close ( $<3$ arcsec) or medium-separation (3-6 arcsec) sources. Figure 6 and Table 2 summarize these results. In a forthcoming paper, we will present a comparison with the speakle and $\mathrm{AO}$ results by Howell et al. (2011) and Adams et al. (2012).
According to all these numbers, we defined the observed companion fraction as $o c f=\frac{n}{s+n}$, where $\mathrm{n}$ is the number of objects within a certain angular separation from the KOI of each particular type of system (either double, triple, or quadruple) and $s$ is the number of isolated KOIs found in our sample (i.e., $s=57$ ). The cumulative $o c f$ is plotted in the upper panel of Fig. 3. We show there that for a given KOI, the probability of having a double (visual) system within 3 arcsec is $21 \%$, while if we go further away until 6 arcsec, this probability increases to $37 \%$. The same was done for triple systems (purple in upper panel of Fig. 3) for which we found that only $3 \%$ of the KOIs have more than 1 visual companion within 3 arcsec. No quadruple systems were detected in our sample within 6 arcsec (orange line in the mentioned figure). We also plot a total observed-companion fraction (black line) taking into account the contributions from all type of systems. This was defined as $o c f($ Total $)=\frac{d+t+q}{s+d+t+q}$, where $d, t$, and $q$ are the double, triple, and quadruple systems found within a certain angular separation, a similar definition as the one presented by Duchêne (1999). We note that in the upper panel of Fig. 3 only the $o c f_{\text {Total }}$ monotonically increases as we move away from the primary. The decreasing cumulative $o c f$ at 7-8 arcsec for the double systems is due to our reaching the position of the third component of a triple system, which is automatically accounted for the triple sample and removed from the double sample. There is a similar explanation for the decreasing cumulative ocf of the triple system.

In Table 5, we present our photometric measurements in the SDSS $i$ and SDSSz bands for KOIs with detected close $(<3$ arcsec) companions. In this table, we also provide the angular separations between the stellar companions and the assumed KOI, as well as the angular position from north towards east. We note that in cases where both objects have similar magnitudes it is impossible with our observations to infer which of the two is actually the one that harbors the transit (high-resolution imaging in- and out-of-transit should be performed to this end). To avoid errors coming from the calibration process, we measured the differential magnitudes $\Delta i$ and $\Delta z$ directly from the instrumental magnitudes. The $i-z$ color was derived directly from the calibrated magnitudes for the primary (brighter) star. To minimize the large errors due to the faintness of the majority of the close companions, we obtained the $(i-z) / \mathrm{C}$ color of the companion by using the expression

$(i-z)_{/ \mathrm{C}}=\Delta i-\Delta z+(i-z)_{P}$,

where $(i-z)_{\mathrm{P}}$ represents the color of the primary star.

Owing to our limited observing time, we only obtained SDSSz images for KOIs found to have very close companions $(<3 \mathrm{arcsec})$ in an on-the-fly reduction. Hence, we lack $i-z$ colors for companions at angular separations of 3-6 arcsec. Photometric and astrometric information available from our observations for these medium-separation sources is shown in Table 6. We note that KOIs with both companions within 36 arcsec and at least one object within $0.3-3$ arcsec, do have SDSSz photometry.

Reconnaissance spectra were acquired for $25 \mathrm{KOIs}$ in order to more accurately derive their spectral types and physical parameters such as temperature and surface gravity. Moreover, as explained in Borucki et al. (2011) this kind of observations is able to reject the possibility of a very close $(<0.2 \operatorname{arcsec})$ binary that would produce a grazing eclipse, since this type of binary would have a radial velocity of tens of $\mathrm{km} / \mathrm{s}$. The data is now being analyzed and will be published in a future paper (Lillo-Box et al., in prep.). 

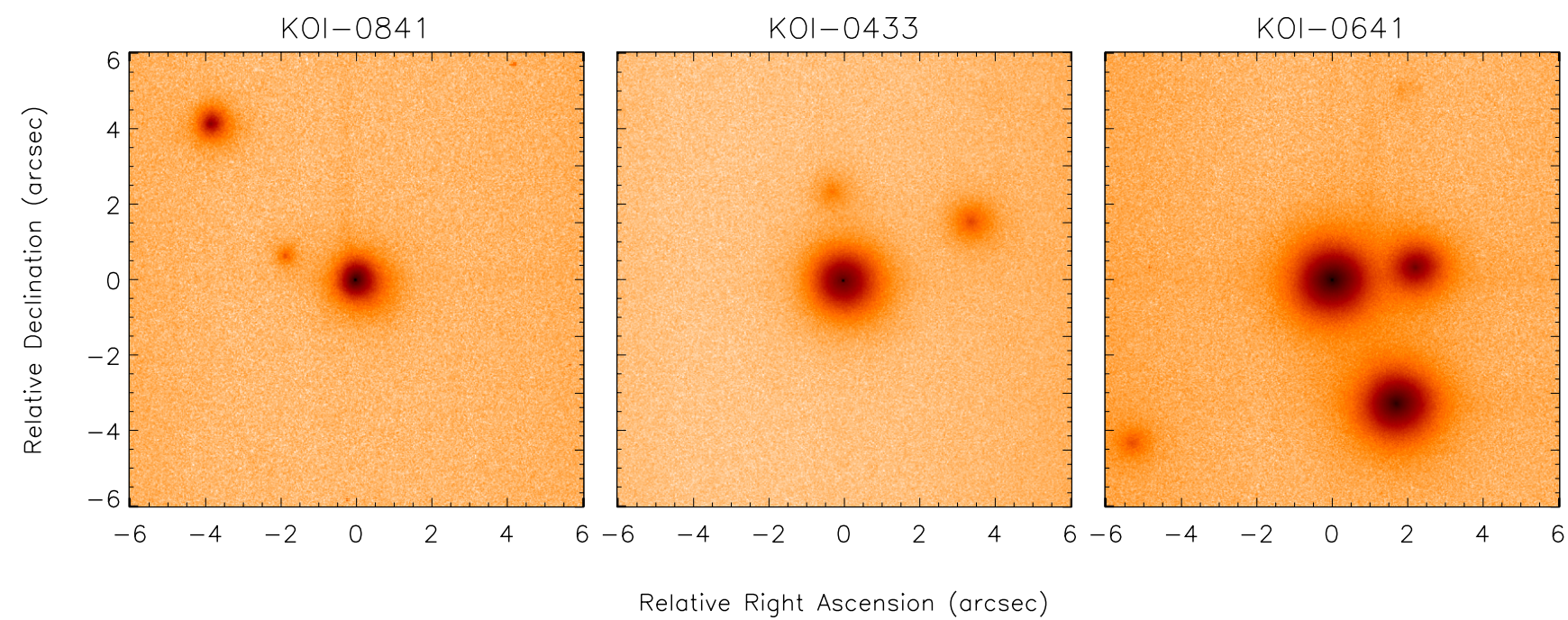

Fig. 4. High-resolution images of the three KOIs with companions in both the 0-3 arcsec and 3-6 arcsec ranges. The images are $12 \times 12$ arcsec and the KOI is placed at the center of the image. Companions are sorted by angular separation in Table 6 . North is up and east is left.

\section{Discussion}

\subsection{Spectral types of the stellar components}

We applied two different methods to determine the spectral type (effective temperature) of the stellar companions according to their angular separation.

\subsubsection{Spectral energy distributions for 3-6 arcsec companions}

Among the 27 KOIs with stellar companions at 3-6 arcsec, we found 2MASS counterparts (Cutri et al. 2003) for around one third of the detected companions (11 out of 30). We assumed an error of 1.5 arcsec for the cross-match between the catalogs. A visual inspection of the 2MASS images was carried out to ensure the accuracy of the match. Together with our SDSSi magnitudes, we performed a spectral energy distribution (SED) fitting with the Virtual Observatory SED Analyzer tool (VOSA, Bayo et al. 2008, and in prep.) to determine the effective temperature of our objects. The new version of this tool allows us to fit the extinction along the line of sight. The results show values for the $A_{v}$ parameter in the range $A_{v}=0-1.0$. Here we assumed a solar metallicity and two surface gravities to account for two possible types of companion at different evolutionary stages: mainsequence foreground or bounded stars $(\log (g)=4.5)$ or background giant stars $(\log (g)=3.5)$. Table 7 summarizes the fit results, according to which these companions are mostly K-type stars if we assume a main-sequence stage. We note that all KOIs are hotter (earlier spectral types) than the possible companions. We found an rms $\sim 200 \mathrm{~K}$ between our fitting results and the KIC effective temperatures. Constraints on the distances for these objects are explained in Sect. 4.2.1.

\subsubsection{Spectral types for close companions}

Assuming a main-sequence nature for the secondary, we were able to estimate their spectral types by using the $i-z$ color. Synthetic spectra from Pickles (1998) for main-sequence stars were convolved with the SDSSi and SDSSz transmission curves 5 following the same scheme as in Daemgen et al. (2009) to derive a relation between the spectral type and the aforementioned $i-z$ color. We overploted our $i-z$ colors in this diagram to estimate the companion spectral types. The typical errors depend on the spectral type since they are determined according to the photometric error in the calculation of the $i-z$ values. In particular, we note that, since the dependence of the spectral type on the aforementioned color is stronger for types later than K5-K6, errors will be much smaller than for earlier types, for which the dependence is weaker. Thus, only stellar companions with $i-z>0.21$ (corresponding to a K5-K6 main-sequence star) are considered in this analysis. In Fig. 7, we show the results for the nine companions and two primaries with estimated spectral types later than K5 (see Table 3).

As expected, these objects are cool, low-mass stars (late K or early M). Their physical association are discussed in Sect. 4.2.

\subsection{On the physical association of the visual companions}

Although the physical bound of the blended stars do not affect our previous calculations, it is important to determine whether the visual companions are, indeed, bounded. Several multiplestar systems have been discovered, both circumbinary planets, for instance Kepler-16b (Doyle et al. 2011), and binary systems with the planet orbiting one of the components of the couple as HD196885Ab (see Thebault 2011). These objects represent a challenge for theoretical models of planet formation, for instance, in terms of orbital stability (see Holman \& Wiegert 1999). Moreover, it has been discovered that around one fifth of known exoplanets inhabit double or multiple stellar systems (Desidera \& Barbieri 2007; Mugrauer \& Neuhäuser 2009; Thebault 2011).

\subsubsection{Medium-distance companions}

We can infer approximate distances to the KOIs and companions analyzed in the previous section by performing a SED fitting

\footnotetext{
5 See

http://www.sdss.org/dr3/instruments/imager/index.html
} 
J. Lillo-Box et al.: Multiplicity in transiting planet-host stars
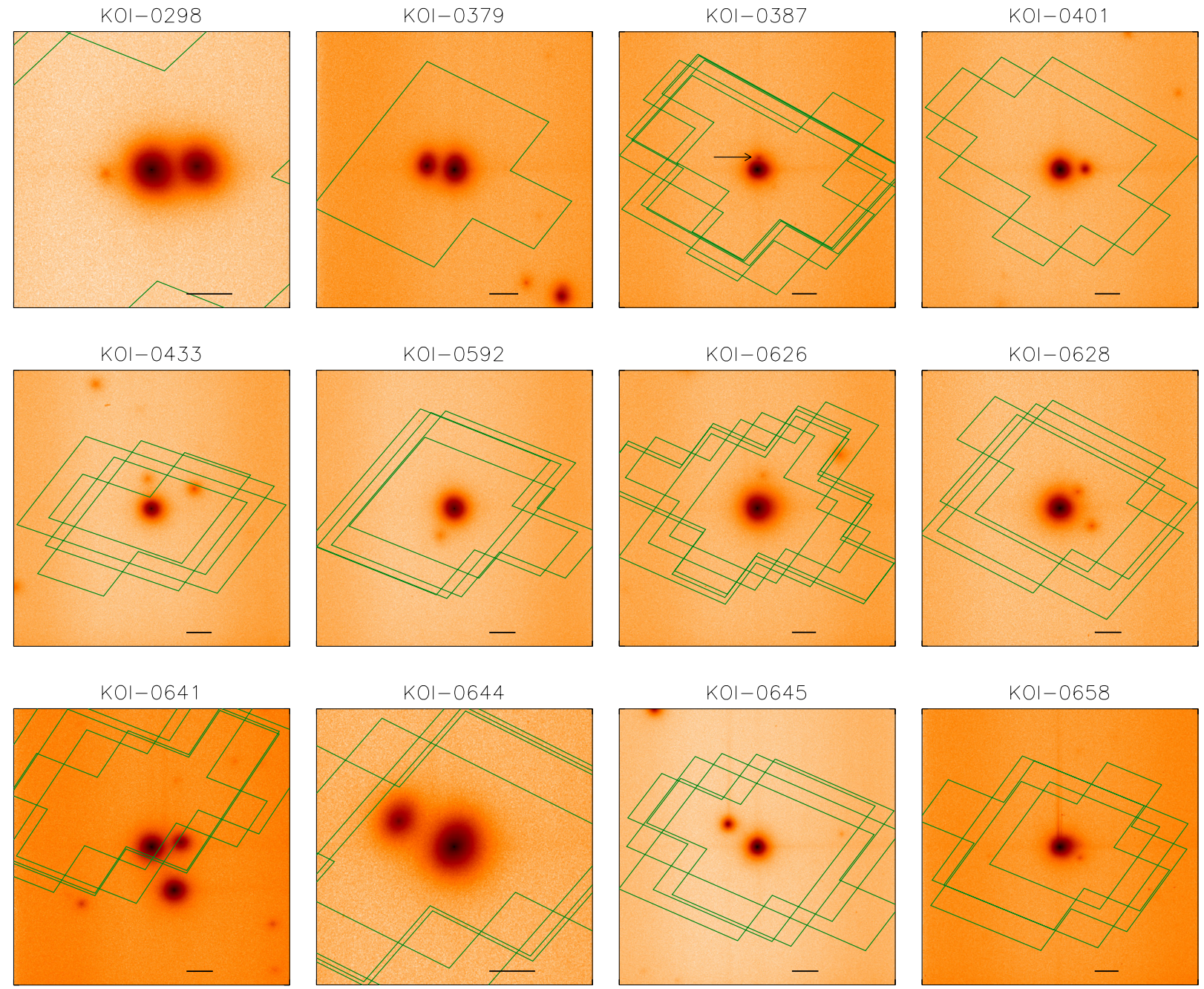

$\mathrm{KOI}-0703$
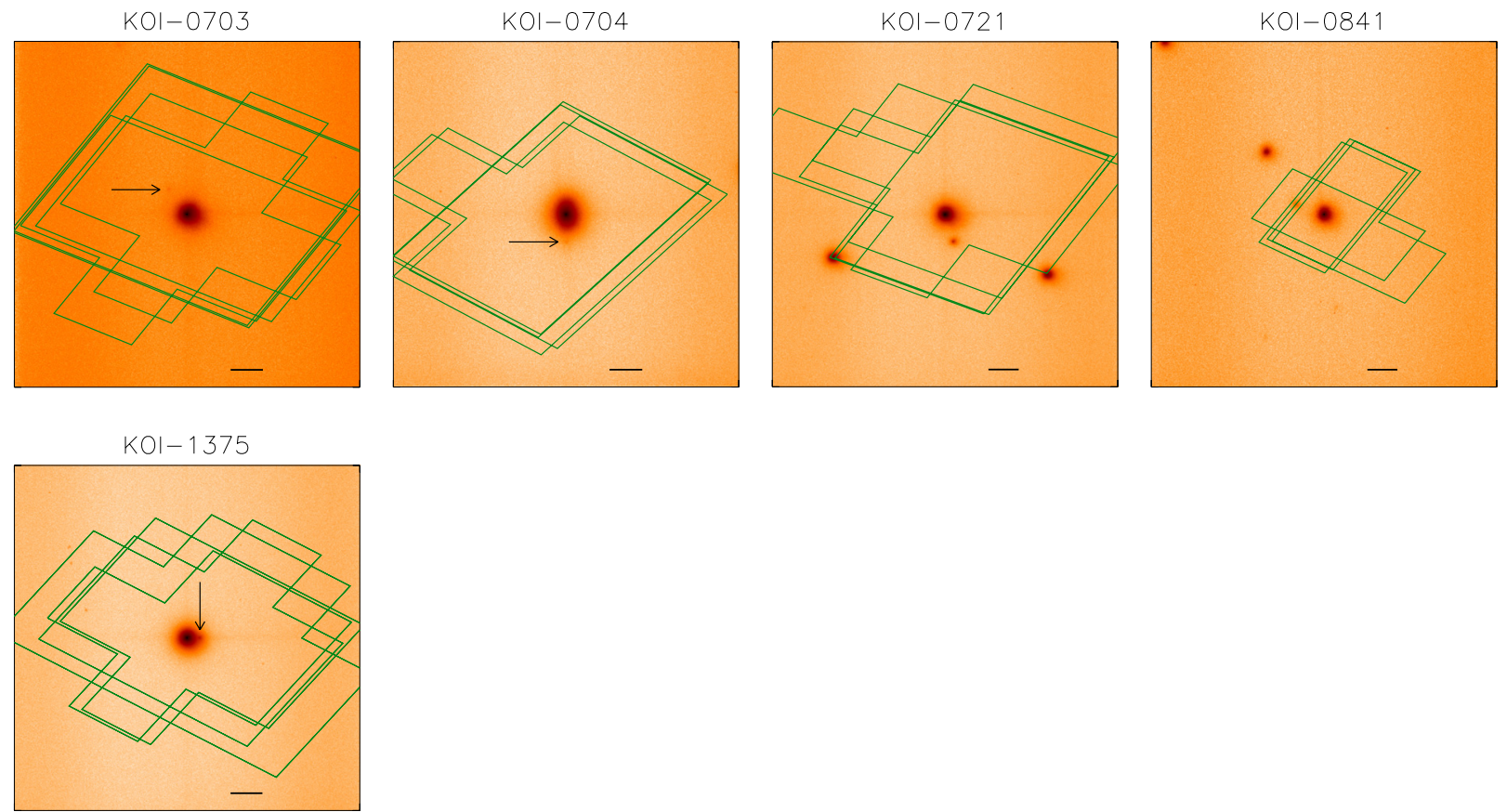

Fig. 5. High-resolution SDSSi images of the close companions detected around 3 arcsec of the 17 Kepler objects of interest. North towards up and east towards left. Different sizes are used for each image to clearly show all features. Black arrows indicate the position of the faintest targets and the horizontal black line represents 2 arcsec on each image. Green polygons represent the optimal public Kepler apertures used on each quarter of the Kepler mission to compute the light curves.The brighter object assumed to be the KOI is centered on the images for reconnaissance purposes. 

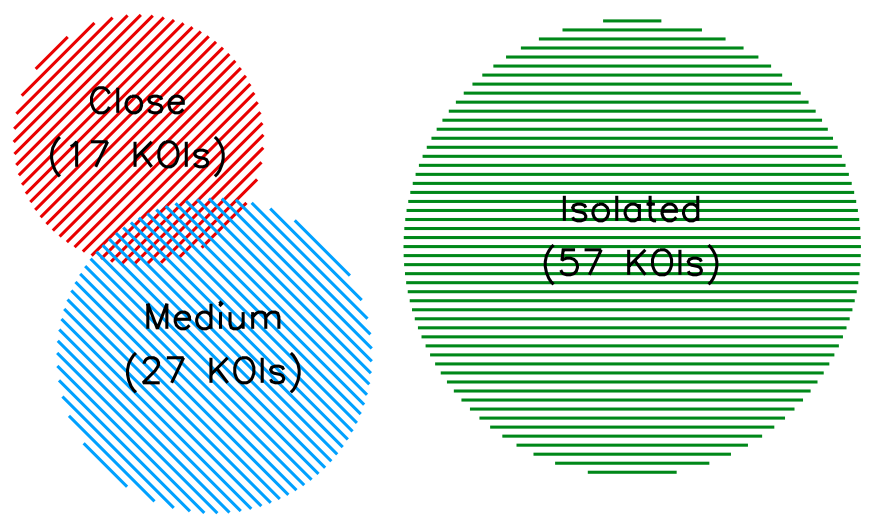

Fig. 6. Main multiplicity results of our survey. Isolated KOIs are represented by the green circle. Kepler objects of interest with objects within 3-6 arcsec are represented by the blue color. The close-companion group (KOIs with at least one source within $0.3-3.0 \mathrm{arcsec}$ ) is shown in red. Note that 3 KOIs (KOI-0433, KOI-0641, and KOI-0841) have objects either in the close- and medium-distance groups.

Table 2. Cumulative percentage of KOIs with visual (even bounded or not) and bound companions according to our observations.

\begin{tabular}{lccc}
\hline \hline & Within 3" & Within 6" & Within 10" \\
\hline Visual & $17.3 \%$ & $41.8 \%$ & $66.3 \%$ \\
Bounded & $6.1-9.2 \%$ & $6.1-9.2 \%$ & - \\
\hline
\end{tabular}

Notes. Visual means here any kind of stellar source detected around the KOI, regardless of whether they are bounded. The bounded sample refers to those objects probably bounded according to the study performed in Sect. 4.2.

using the total flux emitted by the star $\left(F_{\text {tot }}\right)$ according to the effective temperature estimated by VOSA. From this temperature and assuming a luminosity class, we were able to estimate the bolometric magnitude of the star using the relations in Schmidt-Kaler (1982). Hence, we were able to derive a luminosity $L_{*}$ and hence the distance since $L_{*}=4 \pi d^{2} F_{\text {tot }}$. For the KOIs, we assumed a main-sequence stage but two distances were calculated for the stellar companions assuming both mainsequence and giant stages. The errors were calculated by taking into account the uncertainties in $F_{\text {tot }}$ and assuming an error of $125 \mathrm{~K}$ in the temperature calculation for the $L_{*}$ derivation. The former uncertainty also includes the errors in the effective temperature and metallicity fitted by VOSA. The results are shown in the last column of Table 7.

The giant scenario for the companion does not seem to work for most companions since it leads to very large distances ( $d>21 \mathrm{Kpc}$, the Galaxy limit along the Kepler line-of-sight). Thus, the main-sequence scenario is the only possibility for them. According to it, one (KOI-0623C) in ten of the companions that could be fitted by VOSA has a distance in good agreement with its corresponding KOI. Among the remaining eight possible companions, seven are found to be background mainsequence stars and one (KOI-212C) is a probable foreground M-dwarf of spectral type M4V.

\subsubsection{Close companions}

Morton \& Johnson (2011, hereafter MJ11) estimated the probability of a given target in the Kepler field having a background source brighter than $m_{\mathrm{k}}=24.0$ within 2 arcsec, finding that
Table 3. Estimated spectral types for objects with $i-z>0.21$.

\begin{tabular}{lccc}
\hline \hline Object & Lower Limit SpT & SpT & Upper Limit SpT \\
\hline KOI-0641A & K5 & K7 & K9 \\
KOI-0641B & M1 & M3 & M3 \\
KOI-0641C & M3 & M5 & M5 \\
KOI-0703B & K9 & M1 & M3 \\
KOI-0298A & K5 & K7 & K9 \\
KOI-0298B & K9 & M0 & M2 \\
KOI-0658B & M3 & M3 & M3 \\
KOI-0401B & K9 & K9 & M1 \\
KOI-0422B & M5 & M5 & M6 \\
KOI-0645B & K9 & K9 & M1 \\
KOI-0433B & M3 & M5 & M6 \\
\hline
\end{tabular}

Notes. Second and third columns represent the lower and upper limits (respectively) for the spectral type determination according to the method described in the text. Since no decimals have been considered, we estimate an error of one subclass for those with no differences between the error limits and the central value.

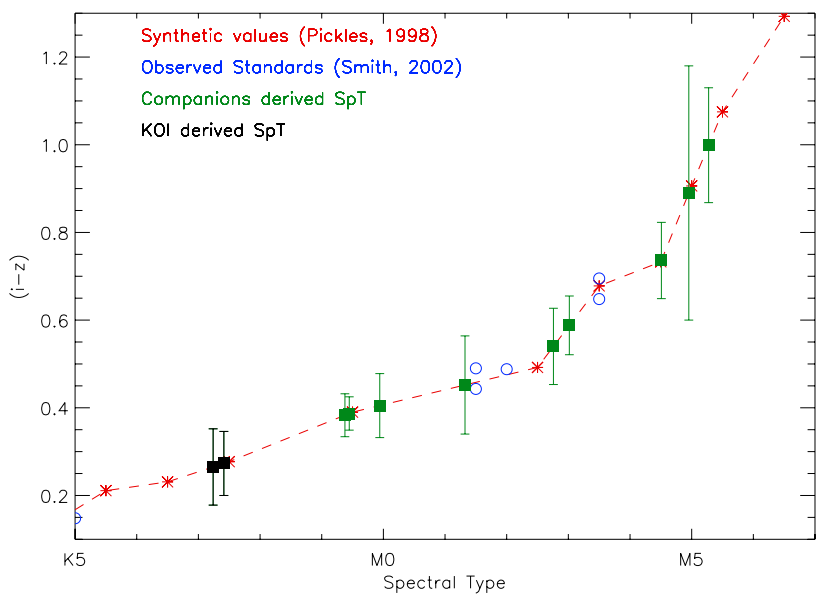

Fig. 7. Spectral type estimation for the stellar companions with $i-z>$ 0.21 corresponding to spectral types later than K5-K6. Red asterisks represent the synthetic values calculated by convolving the spectral library from Pickles (1998) with the transmission curves of the Sloan filters. Blue open circles represent the position of standard stars from Smith et al. (2002). Green filled squares are the measurements for our detected companions and their error bars (assuming no error in the software gain).

this value strongly depends on the Kepler magnitude $\left(m_{\mathrm{k}}\right)$ and Galactic latitude $(b)$ of the target. Since $90 \%$ of our primary targets have Kepler magnitudes in the range 13.0-15.5 and taking into account Eq. (8) in MJ11, we derive a background-source probability-range of 4-40\% depending on the particular values of $b$ and $m_{\mathrm{k}}$ for each target. However, as we have previously said, we reach a completeness of $i=18.5$, which translates into $m_{\mathrm{k}}=18.8$ according to a simple transformation with $r^{2}=0.97$ using both KIC magnitudes. Hence, we underestimate the chance-aligned probability with respect to the one in MJ11. We found 11 blended sources at less than $2 \operatorname{arcsec}$ in 10 parent stars. This means $10.2 \%$ of our sample, which, since it is a lower limit, clearly agrees with the values of MJ11.

Even though our photometric measurements do not provide enough information to clearly establish whether there is a gravitational bound (if any) in the system, we were able to infer some evidence by analyzing several aspects of the available magnitudes of the $\mathrm{A}$ and $\mathrm{B} / \mathrm{C}$ components. We constructed an empirical zero-age main-sequence (ZAMS) based on the synthetic 
J. Lillo-Box et al.: Multiplicity in transiting planet-host stars

Table 4. Observing information of the 41 non-isolated objects in our sample.

\begin{tabular}{|c|c|c|c|c|c|c|c|}
\hline $\begin{array}{l}\text { KOI } \\
\text { ID }\end{array}$ & $\begin{array}{c}\mathrm{RA}^{a} \\
\mathrm{~J} 2000.0\end{array}$ & $\begin{array}{c}\operatorname{Dec}^{a} \\
\mathrm{~J} 2000.0\end{array}$ & Date & $\begin{array}{c}\text { Exptime }^{b} \\
\mathrm{~S}\end{array}$ & Filter & $\begin{array}{c}i_{\text {Complete }} \\
\text { mag }\end{array}$ & $\begin{array}{l}i_{\text {Detect }}{ }^{c} \\
\text { mag }\end{array}$ \\
\hline 99 & $19: 41: 44.23$ & $+44: 31: 52.0$ & 05jul.11 & 60.0 & $\bar{i}$ & 16.34 & 19.94 \\
\hline 131 & $19: 56: 23.42$ & $+43: 29: 51.4$ & 10jul.11 & 150.0 & $i$ & 17.34 & 20.94 \\
\hline 212 & $19: 44: 33.54$ & $+41: 36: 11.5$ & 09jul.11 & 240.0 & $i$ & 17.65 & 21.25 \\
\hline 232 & $19: 24: 26.86$ & $+39: 56: 56.8$ & 02jul.11 & 200.0 & $i$ & 18.40 & 22.00 \\
\hline 238 & $19: 47: 59.68$ & $+42: 46: 55.2$ & 05jul.11 & 200.0 & $i$ & 17.65 & 21.25 \\
\hline 298 & $19: 21: 58.61$ & $+52: 03: 19.8$ & 12jun.11 & 160.0 & $i$ & 17.41 & 21.01 \\
\hline 298 & $19: 21: 58.61$ & $+52: 03: 19.8$ & 12jun.11 & 200.0 & $z$ & - & - \\
\hline 326 & $19: 06: 37.44$ & $+46: 47: 00.6$ & 12jun.11 & 200.0 & $i$ & 18.40 & 22.00 \\
\hline 343 & $19: 40: 28.52$ & $+48: 28: 52.7$ & 12jun.11 & 200.0 & $i$ & 18.40 & 22.00 \\
\hline 372 & $19: 56: 29.40$ & $+41: 52: 00.5$ & 01jul.11 & 34.0 & $i$ & 16.48 & 20.08 \\
\hline 372 & $19: 56: 29.40$ & $+41: 52: 00.5$ & 01jul.11 & 40.0 & $z$ & - & - \\
\hline 375 & $19: 24: 48.28$ & $+51: 08: 39.5$ & 02jul.11 & 87.0 & $i$ & 17.50 & 21.10 \\
\hline 379 & $19: 28: 13.62$ & $+37: 46: 34.3$ & 25jul.11 & 140.0 & $i$ & 17.65 & 21.25 \\
\hline 379 & $19: 28: 13.62$ & $+37: 46: 34.3$ & 25jul.11 & 140.0 & $z$ & - & - \\
\hline 387 & $19: 08: 52.48$ & $+38: 51: 45.0$ & 02jul.11 & 90.0 & $i$ & 17.53 & 21.13 \\
\hline 387 & $19: 08: 52.48$ & $+38: 51: 45.0$ & 02jul.11 & 100.0 & $z$ & - & - \\
\hline 401 & $19: 03: 24.88$ & $+38: 23: 02.8$ & 05jul.11 & 200.0 & $i$ & 18.40 & 22.00 \\
\hline 401 & $19: 03: 24.88$ & $+38: 23: 02.8$ & 05jul.11 & 200.0 & $z$ & - & - \\
\hline 433 & $19: 54: 12.20$ & $+48: 19: 57.0$ & 07jul.11 & 200.0 & $i$ & 17.65 & 21.25 \\
\hline 433 & $19: 54: 12.20$ & $+48: 19: 57.0$ & 07jul.11 & 200.0 & $z$ & - & - \\
\hline 439 & $19: 45: 37.66$ & $+51: 21: 29.5$ & 11jun.11 & 200.0 & $i$ & 18.40 & 22.00 \\
\hline 465 & $19: 35: 42.83$ & $+45: 08: 33.0$ & 10jul.11 & 200.0 & $i$ & 17.65 & 21.25 \\
\hline 520 & $19: 38: 40.31$ & $+43: 51: 11.9$ & 08jul.11 & 200.0 & $i$ & 18.40 & 22.00 \\
\hline 548 & 19:18:00.18 & $+51: 41: 08.5$ & 09jun.11 & 200.0 & $i$ & 18.40 & 22.00 \\
\hline 555 & $19: 32: 29.62$ & $+40: 56: 05.3$ & 10jun.11 & 200.0 & $i$ & 18.40 & 22.00 \\
\hline 592 & $19: 37: 51.02$ & $+46: 49: 17.4$ & 07jul.11 & 200.0 & $i$ & 17.65 & 21.25 \\
\hline 592 & $19: 37: 51.02$ & $+46: 49: 17.4$ & 08jul.11 & 200.0 & $z$ & - & - \\
\hline 611 & $19: 53: 10.57$ & $+41: 41: 01.7$ & 02jul.11 & 200.0 & $i$ & 18.40 & 22.00 \\
\hline 623 & $19: 40: 54.34$ & $+50: 33: 32.4$ & 05jul.11 & 30.0 & $i$ & 16.34 & 19.94 \\
\hline 626 & $19: 40: 46.42$ & $+39: 32: 22.9$ & 04jul.11 & 180.0 & $i$ & 18.09 & 21.69 \\
\hline 626 & $19: 40: 46.42$ & $+39: 32: 22.9$ & 04jul.11 & 180.0 & $z$ & - & - \\
\hline 628 & $19: 14: 47.69$ & $+39: 42: 29.9$ & 04jul.11 & 200.0 & $i$ & 18.40 & 22.00 \\
\hline 628 & $19: 14: 47.69$ & $+39: 42: 29.9$ & 04jul.11 & 160.0 & $z$ & - & - \\
\hline 638 & $19: 42: 14.26$ & $+40: 14: 10.7$ & 05jul.11 & 140.0 & $i$ & 17.65 & 21.25 \\
\hline 641 & $19: 57: 11.88$ & $+40: 14: 06.4$ & 01jul.11 & 87.0 & $i$ & 17.50 & 21.10 \\
\hline 641 & $19: 57: 11.88$ & $+40: 14: 06.4$ & 01jul.11 & 87.0 & $z$ & - & - \\
\hline 644 & $19: 19: 52.03$ & $+40: 31: 57.7$ & 04jul.11 & 180.0 & $i$ & 18.09 & 21.69 \\
\hline 644 & $19: 19: 52.03$ & $+40: 31: 57.7$ & 04jul.11 & 180.0 & $z$ & - & - \\
\hline 645 & $19: 40: 52.18$ & $+40: 35: 32.3$ & 26jun.11 & 200.0 & $i$ & 17.65 & 21.25 \\
\hline 645 & $19: 40: 52.18$ & $+40: 35: 32.3$ & 26jun.11 & 200.0 & $z$ & - & - \\
\hline 658 & $19: 48: 21.60$ & $+41: 23: 16.8$ & 01jul.11 & 200.0 & $i$ & 18.40 & 22.00 \\
\hline 658 & $19: 48: 21.60$ & $+41: 23: 16.8$ & 01jul.11 & 200.0 & $z$ & - & - \\
\hline 685 & $19: 41: 54.17$ & $+43: 29: 35.2$ & 11 jun.11 & 200.0 & $z$ & - & - \\
\hline 685 & $19: 41: 54.17$ & $+43: 29: 35.2$ & 11 jun.11 & 200.0 & $i$ & 18.40 & 22.00 \\
\hline 703 & $19: 39: 38.88$ & $+45: 34: 00.1$ & 11jun.11 & 60.0 & $i$ & 16.89 & 20.49 \\
\hline 703 & $19: 39: 38.88$ & $+45: 34: 00.1$ & 11jun.11 & 200.0 & $z$ & - & - \\
\hline 704 & $18: 57: 32.69$ & $+45: 43: 10.9$ & 05jul.11 & 150.0 & $i$ & 17.65 & 21.25 \\
\hline 721 & $19: 48: 16.42$ & $+46: 50: 03.5$ & 26jun.11 & 140.0 & $i$ & 17.65 & 21.25 \\
\hline 721 & $19: 48: 16.42$ & $+46: 50: 03.5$ & 26jun.11 & 140.0 & $z$ & - & - \\
\hline 841 & $19: 28: 56.82$ & $+41: 05: 09.2$ & 11 jun.11 & 200.0 & $i$ & 18.40 & 22.00 \\
\hline 841 & $19: 28: 56.82$ & $+41: 05: 09.2$ & 11jun.11 & 200.0 & $z$ & - & - \\
\hline 881 & $19: 39: 38.34$ & $+42: 56: 07.1$ & 11 jun.11 & 200.0 & $i$ & 18.40 & 22.00 \\
\hline 1032 & $19: 27: 54.61$ & $+37: 31: 57.4$ & 06jul.11 & 150.0 & $i$ & 18.09 & 21.69 \\
\hline 1192 & $19: 24: 07.70$ & $+38: 42: 14.0$ & 06jul.11 & 200.0 & $i$ & 17.65 & 21.25 \\
\hline 1375 & $19: 13: 16.90$ & $+42: 15: 41.0$ & 06jul.11 & 150.0 & $i$ & 17.34 & 20.94 \\
\hline 1375 & $19: 13: 16.90$ & $+42: 15: 41.0$ & 06jul.11 & 150.0 & $z$ & - & - \\
\hline 1527 & $19: 46: 41.12$ & $+43: 29: 54.2$ & 07jul.11 & 200.0 & $i$ & 17.41 & 21.01 \\
\hline 1573 & $19: 47: 23.06$ & $+40: 08: 19.0$ & 08jul.11 & 200.0 & $i$ & 18.40 & 22.00 \\
\hline 1574 & $19: 51: 40.07$ & $+46: 57: 54.4$ & 06jul.11 & 200.0 & $i$ & 17.65 & 21.25 \\
\hline
\end{tabular}

Notes. ${ }^{(a)}$ Right ascension and Declination from Borucki et al. (2011). ${ }^{(b)}$ Effective exposure time of the image. As the selection rate was of $10 \%$ for all the images, one must multiply this column by 10 to obtain the real exposure time. ${ }^{(c)}$ Estimated completeness and detectability magnitudes scaled to the ones found for the globular cluster M15 (see Sect. 2.3.6) by the exposure time of each particular image. 


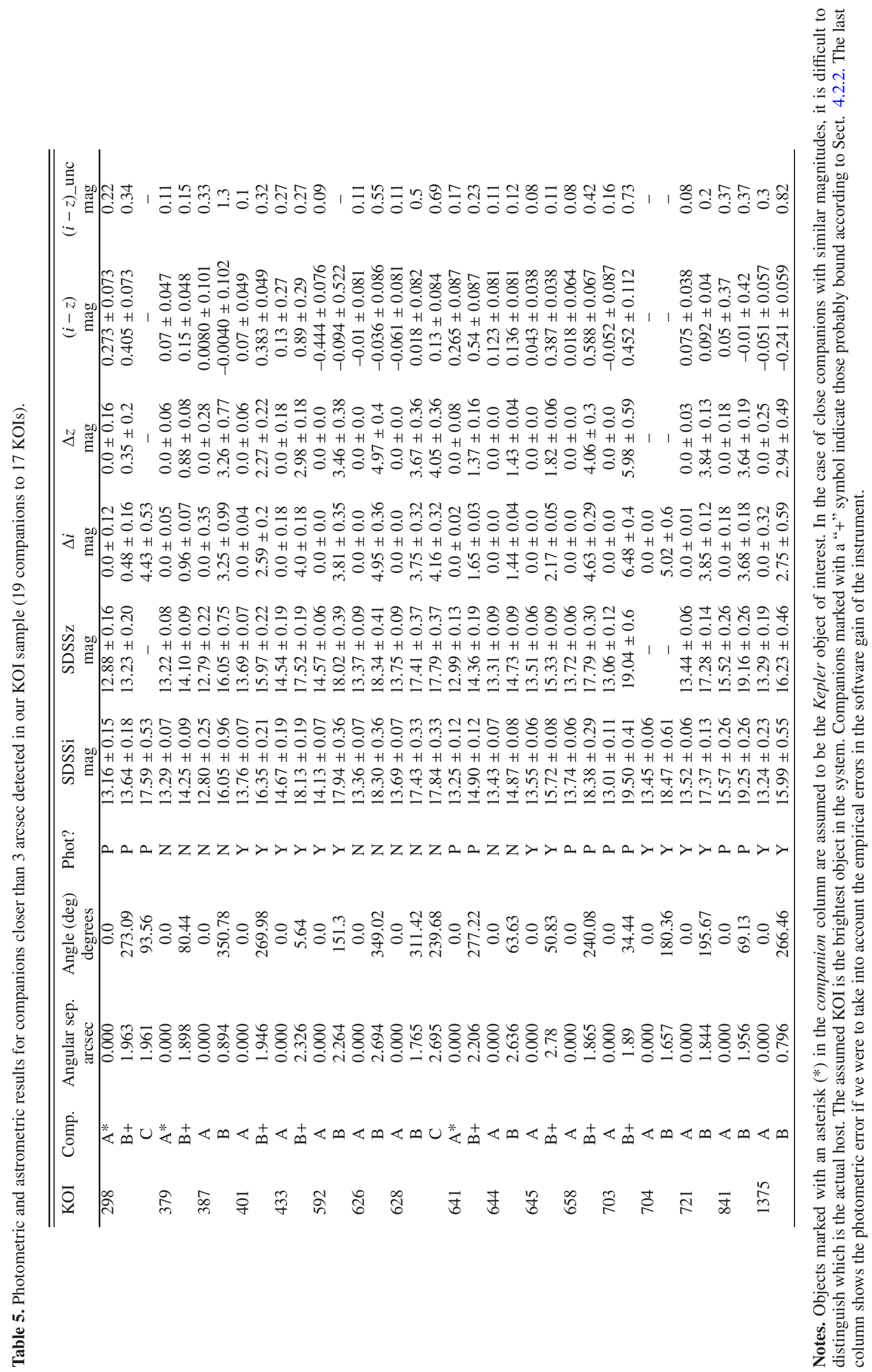


J. Lillo-Box et al.: Multiplicity in transiting planet-host stars

Table 6. Photometric and astrometric results for companions between 3-6 arcsec detected in our KOI sample (30 companions to $27 \mathrm{KOIs).}$

\begin{tabular}{|c|c|c|c|c|c|c|c|c|c|}
\hline KOI & Comp. & $\begin{array}{l}\text { Angular Sep. } \\
\text { arcsec }\end{array}$ & $\begin{array}{c}\text { Angle }(\mathrm{deg}) \\
\text { degrees }\end{array}$ & Phot? & $\begin{array}{l}\text { SDSSi } \\
\text { mag }\end{array}$ & $\begin{array}{l}\text { SDSSz } \\
\text { mag }\end{array}$ & $\begin{array}{c}\Delta i \\
\operatorname{mag}\end{array}$ & $\begin{array}{c}\Delta z \\
\operatorname{mag}\end{array}$ & $\begin{array}{l}(i-z) \\
\text { mag }\end{array}$ \\
\hline 99 & $\mathrm{~A}$ & 0.000 & 0.0 & $\bar{Y}$ & $12.57 \pm 0.18$ & - & $0.00 \pm 0.18$ & - & - \\
\hline 99 & B & 3.383 & 49.3 & Y & $17.96 \pm 0.18$ & - & $5.39 \pm 0.19$ & - & - \\
\hline 99 & $\mathrm{C}$ & 5.997 & 338.3 & Y & $16.58 \pm 0.18$ & - & $4.01 \pm 0.18$ & - & - \\
\hline 131 & A & 0.000 & 0.0 & Y & $13.53 \pm 0.17$ & - & $0.00 \pm 0.18$ & - & - \\
\hline 131 & B & 5.540 & 154.6 & Y & $17.00 \pm 0.17$ & - & $3.48 \pm 0.18$ & - & - \\
\hline 212 & A & 0.000 & 0.0 & $\mathrm{P}$ & $14.70 \pm 0.19$ & - & $0.00 \pm 0.18$ & - & - \\
\hline 212 & B & 4.812 & 308.8 & $\mathrm{P}$ & $17.52 \pm 0.19$ & - & $2.82 \pm 0.18$ & - & - \\
\hline 232 & A & 0.000 & 0.0 & $\mathrm{~N}$ & $13.76 \pm 0.16$ & - & $0.00 \pm 0.18$ & - & - \\
\hline 232 & B & 5.550 & 22.3 & $\mathrm{~N}$ & $18.19 \pm 0.16$ & - & $4.43 \pm 0.18$ & - & - \\
\hline 238 & A & 0.000 & 0.0 & Y & $13.74 \pm 0.18$ & - & $0.00 \pm 0.18$ & - & - \\
\hline 238 & B & 3.769 & 328.7 & Y & $20.61 \pm 0.24$ & - & $6.87 \pm 0.24$ & - & - \\
\hline 326 & A & 0.000 & 0.0 & $\mathrm{P}$ & $14.84 \pm 0.26$ & - & $0.00 \pm 0.18$ & - & - \\
\hline 326 & B & 3.452 & 269.4 & $\mathrm{P}$ & $16.61 \pm 0.26$ & - & $1.77 \pm 0.18$ & - & - \\
\hline 343 & A & 0.000 & 0.0 & $\mathrm{P}$ & $13.06 \pm 0.26$ & - & $0.00 \pm 0.18$ & - & - \\
\hline 343 & B & 4.938 & 145.8 & $\mathrm{P}$ & - & - & - & - & - \\
\hline 372 & A & 0.000 & 0.0 & $\mathrm{P}$ & $12.25 \pm 0.18$ & $12.12 \pm 0.18$ & $0.00 \pm 0.18$ & $0.00 \pm 0.18$ & $0.13 \pm 0.25$ \\
\hline 372 & B & 5.962 & 36.1 & $\mathrm{P}$ & $16.88 \pm 0.18$ & $16.58 \pm 0.18$ & $4.64 \pm 0.18$ & $4.45 \pm 0.18$ & $0.30 \pm 0.25$ \\
\hline 375 & A & 0.000 & 0.0 & $\mathrm{~N}$ & $13.08 \pm 0.15$ & - & $0.00 \pm 0.18$ & - & - \\
\hline 375 & B & 5.329 & 156.1 & $\mathrm{~N}$ & $18.06 \pm 0.16$ & - & $4.98 \pm 0.18$ & - & - \\
\hline 433 & A & 0.000 & 0.0 & Y & $14.67 \pm 0.19$ & $14.54 \pm 0.19$ & $0.00 \pm 0.18$ & $0.00 \pm 0.18$ & $0.13 \pm 0.27$ \\
\hline 433 & $\mathrm{C}$ & 3.670 & 293.4 & Y & $17.26 \pm 0.19$ & $17.07 \pm 0.19$ & $2.60 \pm 0.18$ & $2.53 \pm 0.18$ & $0.19 \pm 0.27$ \\
\hline 439 & A & 0.000 & 0.0 & $\mathrm{P}$ & $14.11 \pm 0.32$ & - & $0.00 \pm 0.18$ & - & - \\
\hline 439 & $\mathrm{~B}$ & 5.453 & 16.9 & $\mathrm{P}$ & $19.45 \pm 0.32$ & - & $5.34 \pm 0.19$ & - & - \\
\hline 465 & A & 0.000 & 0.0 & Y & $13.94 \pm 0.17$ & - & $0.00 \pm 0.18$ & - & - \\
\hline 465 & B & 3.580 & 130.8 & $\mathrm{Y}$ & $18.30 \pm 0.17$ & - & $4.35 \pm 0.18$ & - & - \\
\hline 465 & $\mathrm{C}$ & 4.527 & 193.1 & Y & $17.82 \pm 0.17$ & - & $3.87 \pm 0.18$ & - & - \\
\hline 520 & A & 0.000 & 0.0 & $\mathrm{~N}$ & $14.30 \pm 0.17$ & - & $0.00 \pm 0.18$ & - & - \\
\hline 520 & B & 5.628 & 271.7 & $\mathrm{~N}$ & $18.14 \pm 0.17$ & - & $3.84 \pm 0.18$ & - & - \\
\hline 548 & A & 0.000 & 0.0 & $\mathrm{~N}$ & $13.37 \pm 0.50$ & - & $0.00 \pm 0.18$ & - & - \\
\hline 548 & B & 4.597 & 133.9 & $\mathrm{~N}$ & $18.33 \pm 0.50$ & - & $4.96 \pm 0.18$ & - & - \\
\hline 555 & A & 0.000 & 0.0 & $\mathrm{~N}$ & $14.29 \pm 0.18$ & - & $0.00 \pm 0.18$ & - & - \\
\hline 555 & B & 4.021 & 23.1 & $\mathrm{~N}$ & $17.86 \pm 0.18$ & - & $3.58 \pm 0.18$ & - & - \\
\hline 611 & A & 0.000 & 0.0 & $\mathrm{~N}$ & $13.75 \pm 0.15$ & - & $0.00 \pm 0.18$ & - & - \\
\hline 611 & B & 5.999 & 324.2 & $\mathrm{~N}$ & $19.18 \pm 0.16$ & - & $5.44 \pm 0.18$ & - & - \\
\hline 623 & A & 0.000 & 0.0 & Y & $11.55 \pm 0.18$ & - & $0.00 \pm 0.18$ & - & - \\
\hline 623 & B & 5.540 & 202.0 & Y & $14.07 \pm 0.18$ & - & $2.52 \pm 0.18$ & - & - \\
\hline 638 & A & 0.000 & 0.0 & Y & $13.51 \pm 0.17$ & - & $0.00 \pm 0.18$ & - & - \\
\hline 638 & $\mathrm{~B}$ & 5.938 & 65.5 & Y & $19.43 \pm 0.19$ & - & $5.92 \pm 0.19$ & - & - \\
\hline 641 & A & 0.000 & 0.0 & $\mathrm{P}$ & $13.25 \pm 0.12$ & $12.99 \pm 0.13$ & $0.00 \pm 0.02$ & $0.00 \pm 0.08$ & $0.27 \pm 0.17$ \\
\hline 641 & $\mathrm{C}$ & 3.653 & 206.1 & $\mathrm{P}$ & $13.38 \pm 0.16$ & $12.89 \pm 0.17$ & $0.29 \pm 0.18$ & $0.08 \pm 0.18$ & $0.49 \pm 0.23$ \\
\hline 685 & A & 0.000 & 0.0 & $\mathrm{P}$ & $13.71 \pm 0.3$ & - & $0.00 \pm 0.18$ & - & - \\
\hline 685 & B & 3.268 & 271.1 & $\mathrm{P}$ & $19.24 \pm 0.3$ & - & $5.54 \pm 0.18$ & - & - \\
\hline 841 & A & 0.000 & 0.0 & $\mathrm{P}$ & $15.57 \pm 0.26$ & $15.52 \pm 0.26$ & $0.00 \pm 0.18$ & $0.00 \pm 0.18$ & $0.05 \pm 0.37$ \\
\hline 841 & $\mathrm{C}$ & 5.599 & 41.5 & $\mathrm{P}$ & $17.19 \pm 0.26$ & $17.20 \pm 0.26$ & $1.62 \pm 0.18$ & $1.68 \pm 0.18$ & $-0.01 \pm 0.37$ \\
\hline 881 & A & 0.000 & 0.0 & $\mathrm{P}$ & $15.58 \pm 0.26$ & - & $0.00 \pm 0.18$ & - & - \\
\hline 881 & B & 5.264 & 191.5 & $\mathrm{P}$ & $20.70 \pm 0.29$ & - & $5.12 \pm 0.22$ & - & - \\
\hline 1032 & A & 0.000 & 0.0 & Y & $13.47 \pm 0.17$ & - & $0.00 \pm 0.18$ & - & - \\
\hline 1032 & B & 5.856 & 320.3 & Y & $18.90 \pm 0.17$ & - & $5.43 \pm 0.18$ & - & - \\
\hline 1032 & $\mathrm{C}$ & 5.883 & 86.1 & Y & $18.42 \pm 0.17$ & - & $4.94 \pm 0.18$ & - & - \\
\hline 1192 & A & 5.643 & 195.1 & Y & $12.52 \pm 0.17$ & - & $0.00 \pm 0.18$ & - & - \\
\hline 1192 & $\mathrm{~B}$ & 0.000 & 0.0 & Y & $17.82 \pm 0.17$ & - & $5.30 \pm 0.18$ & - & - \\
\hline 1527 & A & 0.000 & 0.0 & Y & $14.73 \pm 0.19$ & - & $0.00 \pm 0.18$ & - & - \\
\hline 1527 & B & 5.908 & 346.9 & Y & $17.40 \pm 0.19$ & - & $2.67 \pm 0.18$ & - & - \\
\hline 1573 & A & 0.000 & 0.0 & $\mathrm{~N}$ & $14.17 \pm 0.17$ & - & $0.00 \pm 0.18$ & - & - \\
\hline 1573 & B & 3.933 & 300.1 & $\mathrm{~N}$ & $18.84 \pm 0.17$ & - & $4.68 \pm 0.18$ & - & - \\
\hline 1574 & A & 0.000 & 0.0 & Y & $14.26 \pm 0.16$ & - & $0.00 \pm 0.18$ & - & - \\
\hline 1574 & $\mathrm{~B}$ & 5.035 & 224.6 & $\mathrm{Y}$ & $18.92 \pm 0.17$ & - & $4.66 \pm 0.18$ & - & - \\
\hline
\end{tabular}

Notes. Only objects with additional companions within 3 arcsec have magnitudes in the SDSSz. KOI-0372 also has this information because during the observing run it had seemed to have a close companion that, however, after the reduction was discarded.

griz photometry derived by Ofek (2008) for Tycho-2 stars with HIPPARCOS $B_{\mathrm{T}}$ and $V_{\mathrm{T}}$ bands, as well as 2MASS $J H K$ magnitudes (see Fig. 8). To reach the substellar domain, we added the Moraux et al. (2003) iz photometry for 109 brown dwarfs in the Pleiades region assuming a distance to the cluster of $130 \mathrm{pc}$ (Stello \& Nissen 2001). As we are interested in possible binary 
A\&A 546, A10 (2012)

Table 7. Spectral-energy-distribution fitting results for the medium-distance companions with 2MASS counterparts.

\begin{tabular}{|c|c|c|c|c|c|c|c|c|c|}
\hline KOI & Comp. & $\mathrm{RA}^{a}$ & $\operatorname{Dec}^{a}$ & $T_{\text {eff }}$ & $\log (g)$ & $\chi^{2}$ & SpT & Model $^{b}$ & $D(\mathrm{pc})$ \\
\hline \multirow[t]{3}{*}{131} & A & 299.097600 & 43.497601 & 6500.0 & 4.5 & 0.47 & F5 & Kurucz & $1120_{-120}^{+100}$ \\
\hline & B & 299.098259 & 43.496210 & 5500.0 & 4.5 & 0.90 & G8 & Kurucz & $2510_{-350}^{+330}$ \\
\hline & B & 299.098259 & 43.496210 & 5400.0 & 3.5 & 0.86 & G3 & NextGen & $-{ }^{d}$ \\
\hline \multirow[t]{5}{*}{212} & A & 296.139750 & 41.603199 & 5800.0 & 4.5 & 0.92 & G4 & NextGen & $1010_{-130}^{+230}$ \\
\hline & B & 296.138708 & 41.604036 & 4400.0 & 4.5 & 5.66 & K5 & NextGen & $1760_{-240}^{+230}$ \\
\hline & $\mathrm{C}$ & 296.139026 & 41.601652 & 3400.0 & 4.5 & 0.61 & M4 & COND00 & $366_{-94}^{+136}$ \\
\hline & B & 296.138708 & 41.604036 & 4200.0 & 3.5 & 5.55 & K3 & NextGen & $-^{d}$ \\
\hline & $\mathrm{C}$ & 296.139026 & 41.601652 & 3100.0 & 3.5 & 2.34 & $>\mathrm{M} 6$ & COND00 & $-^{c}$ \\
\hline \multirow[t]{3}{*}{372} & A & 299.122500 & 41.866798 & 5750.0 & 4.5 & 1.21 & G5 & Kurucz & $314_{-38}^{+85}$ \\
\hline & B & 299.123475 & 41.868137 & 4600.0 & 4.5 & 0.68 & K4 & NextGen & $1340_{-180}^{+230}$ \\
\hline & B & 299.123475 & 41.868137 & 4400.0 & 3.5 & 0.67 & $\mathrm{~K} 2$ & NextGen & $-^{d}$ \\
\hline \multirow[t]{3}{*}{375} & A & 291.201150 & 51.144299 & 5800.0 & 4.5 & 1.60 & G4 & NextGen & $495_{-65}^{+112}$ \\
\hline & B & 291.201750 & 51.142945 & 3500.0 & 4.5 & 1.41 & M3 & NextGen & $831_{-212}^{+123}$ \\
\hline & B & 291.201750 & 51.142945 & 3100.0 & 3.5 & 3.04 & $>\mathrm{M} 6$ & COND00 & $-^{c}$ \\
\hline \multirow[t]{3}{*}{520} & A & 294.667950 & 43.853298 & 5200.0 & 4.5 & 5.36 & K0 & NextGen & $567_{-60}^{+66}$ \\
\hline & B & 294.666387 & 43.853345 & 3600.0 & 4.5 & 14.80 & M2 & DUSTY00 & $1020_{-130}^{+170}$ \\
\hline & B & 294.666387 & 43.853345 & 3900.0 & 3.5 & 15.70 & K6 & COND00 & $-{ }^{d}$ \\
\hline \multirow[t]{3}{*}{623} & A & 295.226400 & 50.558998 & 6250.0 & 4.5 & 1.16 & F7 & Kurucz & $411_{-60}^{+59}$ \\
\hline & B & 295.225822 & 50.557572 & 4800.0 & 4.5 & 2.41 & $\mathrm{~K} 3$ & NextGen & $439_{-55}^{+47}$ \\
\hline & B & 295.225822 & 50.557572 & 4400.0 & 3.5 & 1.88 & $\mathrm{~K} 2$ & NextGen & $7920_{-1300}^{+960}$ \\
\hline \multirow[t]{3}{*}{641} & A & 299.299500 & 40.235100 & 3600.0 & 4.5 & 1.29 & M2 & DUSTY00 & $117_{-15}^{+19}$ \\
\hline & $\mathrm{C}$ & 299.299054 & 40.234189 & 3400.0 & 4.5 & 1.60 & M4 & NextGen & $76_{-20}^{+28}$ \\
\hline & $\mathrm{C}$ & 299.299054 & 40.234189 & 3700.0 & 3.5 & 1.68 & M1 & COND00 & $10370_{-2400}^{+1800}$ \\
\hline \multirow[t]{3}{*}{841} & A & 292.236750 & 41.085899 & 5400.0 & 4.5 & 1.17 & G9 & NextGen & $1170_{-150}^{+160}$ \\
\hline & $\mathrm{C}$ & 292.237780 & 41.087064 & 5000.0 & 4.5 & 1.53 & K1 & NextGen & $2120_{-270}^{+1070}$ \\
\hline & $\mathrm{C}$ & 292.237780 & 41.087064 & 4800.0 & 3.5 & 1.46 & G9 & NextGen & $10830_{-1900}^{+1900}$ \\
\hline \multirow[t]{3}{*}{1527} & A & 296.671350 & 43.498402 & 5400.0 & 4.5 & 1.56 & G9 & NextGen & $773_{-98}^{+103}$ \\
\hline & B & 296.670978 & 43.500000 & 4800.0 & 4.5 & 2.08 & K3 & NextGen & $1980_{-220}^{+220}$ \\
\hline & $\mathrm{B}$ & 296.670978 & 43.500000 & 4600.0 & 3.5 & 1.90 & K1 & NextGen & $-^{d}$ \\
\hline
\end{tabular}

Notes. ${ }^{(a)}$ Calculated RA and Dec from the position of the KOI according to the distance and angle derived in this paper. ${ }^{(b)}$ Best fit model used by VOSA. See http://svo.cab.inta-csic.es/theory/vosa/ for more information. ${ }^{(c)}$ Effective temperature is outside the model range (d) Estimated distance is greater than $21 \mathrm{Kpc}$ (the estimated Milky Way limit in the Kepler line of sight).

stars, we computed the lower envelope of the ZAMS for both catalogs together in an $i$ vs. $i-z$ color-magnitude diagram. Primary objects were located in the empirical ZAMS according to their $i-z$ color, which was calculated from our own photometry. Hence, a distance modulus was calculated for the primary star and applied to the secondary star assuming the joint formation of both stars. Figure 8 shows the results for all of our 17 KOIs with their 19 close stellar companions. The data for at least 6 companions agree with a simultaneous formation of stars in a double system (KOIs 0379B, 0645B, 0298B, 0641B, 0658B, and 0401B), together with the KOI star. Another three close objects could also lie within the ZAMS boundaries if we take into account the errors in the determination of this isochrone
(KOIs 0592B, 0433B and 0703B). We also note that some of them lie in the low-mass domain of the diagram, implying that KOI-0641B, KOI-0658B, KOI-0433B, and KOI-0703B could be either low-mass stars or even brown-dwarf companions to the host stars.

Brandner et al. (2000) provided a simple formula to estimate the probability that a given source at a certain angular separation to be a background object. If we assume the limiting magnitude of the USNO-B1.06 catalog (Monet et al. 2003) in the Kepler field to be $I=17.5 \mathrm{mag}$, we find an overall probability of $3.9 \%$ that a source is separated 3 arcsec from our target

6 United States Naval Observatory. 


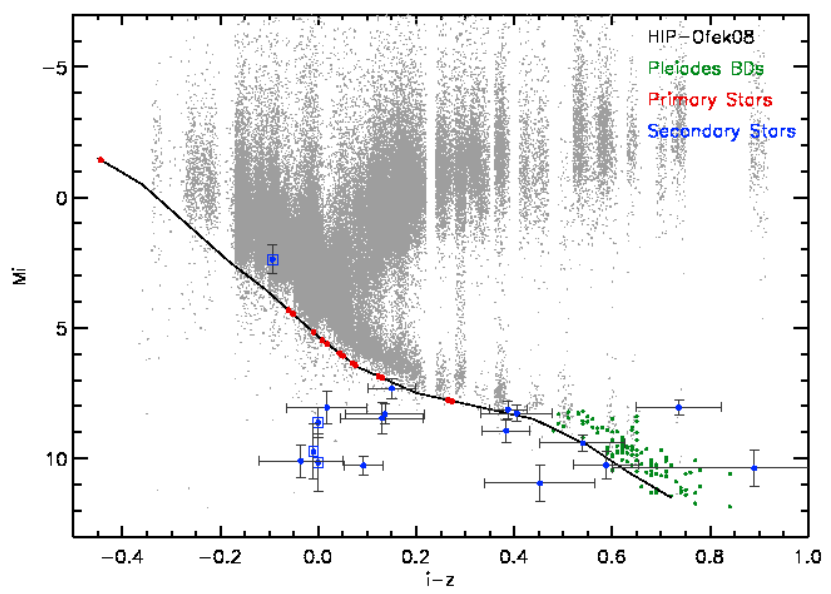

Fig. 8. Empirical ZAMS (solid black line) computed using synthetic $i z$ photometry from Ofek (2008), grey dots, and Moraux et al. (2003), green dots. Primary objects are represented by red filled circles and secondary companions with blue filled circles. Companions with large errors in their $i-z$ colors are plotted with blue open squares.

to be a background object. This shows, although as a first approximation, the low probability of our close companions being background sources rather than bound binaries.

Our analysis implies that 6-9 of our identified companions out of the 98 KOIs observed could be physically bound. This means an observational lower limit of $6.2 \%-9.2 \%$ for the binarity rate among the Kepler targets (the presence of binaries with projected separations or magnitudes or both below our detection limits may increase these values). However, we caution that this result may be unreliable owing to the lack of confirmation of their physical bond. We have instead shown several pieces of evidence that point to this scenario. As we have said before, it has been estimated that $20 \%$ of planets reside in binary systems that are mostly wide binaries with separations greater than 1000 AU (Thebault 2011; Desidera \& Barbieri 2007; Mugrauer $\&$ Neuhäuser 2009). This would be in good agreement with our results if the physical bound were confirmed for the 6-9 KOIs mentioned above. Such widely separated companions would have weakly affected the planet formation. Desidera \& Barbieri (2007) concluded that companions at separations of more than 100-300 AU would not significantly influence the formation and migration of planets and thus that planet parameters may not have relevant differences from those inhabiting single stars, except for an overabundance of high eccentricity orbits in the wide-binary planets. We do not detect any differences between the KIC-derived physical and orbital parameters of the 6-9 KOIs and those of the rest of the observed sample, confirming the results previously mentioned.

We note that our estimated binary fraction is a lower limit owing to our observational restrictions. Close and/or faint (lowmass) companions beyond our detectable limits (described in Sect. 2.3.6) might increase this multiplicity fraction. Raghavan et al. (2010) obtained a binary frequency of $34 \%$ for companions around close $(<35 \mathrm{pc})$ solar-like stars in the magnitude range $V=4-12$. Although our present survey encompasses a different range of stellar masses, their result suggests that our study might indeed be missing a number of close and faint companions.

\subsection{Need for update of planet-star parameters}

The incidence among the planet and host-star parameters of either bound or visual companions to the host stars can be measured by isolating the contribution of the host star from the blended companion. Although the transit depth defined as $F_{\text {notransit }}-F_{\text {transit }}$ does not depend on the presence of blended objects in the PSF, the physical properties of the planet-star system actually depend on the quotient $\Delta F=\left(F_{\text {notransit }}-F_{\text {transit }}\right) / F_{\text {notransit }}$ as shown by Seager \& Mallén-Ornelas (2003). This quantity clearly decreases with the closeness of a blended object since it would contribute an additional flat flux (an intrinsic variability of the blended star would be visible in the light curve). Hence, a change in $\left(F_{\text {notransit }}-F_{\text {blend }}\right) / F_{\text {notransit }}$ will lead to consequent changes in the planet-star parameters. Assuming that the planet is actually transiting the brighter star, the new depth will be given by (see Daemgen et al. 2009)

$\Delta F_{\text {new }}=\left(1+10^{-\frac{\Delta z}{2.5}}\right) \Delta F_{\text {old }}$

We note that for the two systems where more than one blended object is found, the $\Delta z$ should be computed as

$z_{/ \mathrm{C}}^{\prime}=-2.5 \log \left[\sum_{j} 10^{-0.4 z_{j}}\right]$.

Additional photometric studies such as on-transit and out-oftransit high-resolution images of the fainter star as well as observations of the transit depth at longer wavelengths, such as with the IRAC bands of the Spitzer Space Telescope (see, for example, Desert et al. 2011), would be needed to rule out the possibility of the planet transiting the fainter star.

The impact on the physical parameters of a blended companion could be quite significant. The largest change in the transit depth values according to Eq. (6) is $\Delta F_{\text {new }} \leq \sqrt{2} \Delta F_{\text {old }}$, assuming an equal-magnitude blended star. According to Eq. (6) in Seager \& Mallén-Ornelas (2003), this would increase the planet-to-star radius ratio by $41 \%$. The same calculation can be done for other parameters such us the total on-transit duration $\left(t_{\mathrm{T}}\right)$, total on-transit flat duration $\left(t_{\mathrm{F}}\right)$, and period $(P)$. We performed this exercise for our observations. Table 8 shows the recalculated values of the transit depth, planet-to-star radius ratio, and planet radius for the 24 planets orbiting the 17 stars with close companions. The last two parameters were calculated by assuming no-limb darkening, using the aforementioned formula in Seager \& Mallén-Ornelas (2003) of: $R_{\mathrm{p}} / R_{*}=\sqrt{\Delta F}$. Our results show the relative differences in the transit depths, calculated as $\left(\delta_{\text {new }}-\delta_{\text {cat }}\right) / \delta_{\text {cat }}$, in the range $1 \%-120 \%$ from the values published by Batalha et al. (2012). According to the expression explained above, $74 \%$ of the planets with detected companions at separations of less than 3 arcsec would have changes in their $R_{\mathrm{p}} / R_{\mathrm{s}}$ parameter below $10 \%$, and for $91 \%$ of them below $30 \%$.

\subsection{Particular cases}

\subsubsection{KOI-0641}

This system is of particular interests because it is a triple visual system of size 3.7 arcsec. Its $i-z$ colors correspond to spectral types of K5-K9, M1-M3, and M3-M5 for the A, B, and C components, respectively. Regarding the SED fitting, we obtained the spectral types M2 and M3-M4 for the A and C components, respectively. The distances to the primary target for both approaches agree: they are $d=125 \pm 22 \mathrm{pc}$ and $d=117_{-5}^{+6} \mathrm{pc}$. The KOI-0641B system is perfectly consistent with the bound scenario according to its position in the ZAMS. If this scenario indeed were the case, the system would have a projected distance of $275 \pm 49$ AU from the A component. The third object is 
Table 8. New planet-transit depth and relative radii to the parent star accounting for the blended objects in the Kepler images.

\begin{tabular}{lccccccc}
\hline \hline $\begin{array}{l}\text { Planet ID } \\
(\text { KOI.Planet })\end{array}$ & $\begin{array}{c}\text { Cat. Depth } \\
(\mathrm{ppm})\end{array}$ & $\begin{array}{c}\text { NewDepth } \\
(\mathrm{ppm})\end{array}$ & $\begin{array}{c}\text { Cat } R_{\mathrm{p}} / R_{*} \\
\left(10^{-2}\right)\end{array}$ & $\begin{array}{c}\text { New } R_{\mathrm{p}} / R_{*}{ }^{a} \\
\left(10^{-2}\right)\end{array}$ & $\begin{array}{c}\text { Sec. } R_{\mathrm{p}} / R_{*}{ }^{b} \\
\left(10^{-2}\right)\end{array}$ & $\begin{array}{c}\text { Cat. } R_{\mathrm{p}}{ }^{c} \\
R_{\text {Earth }}\end{array}$ & $\begin{array}{c}\text { New } R_{\mathrm{p}}{ }^{d} \\
R_{\text {Earth }}\end{array}$ \\
\hline 298.01 & 274 & $472 \pm 67$ & $1.41 \pm 0.04$ & $2.2 \pm 0.2$ & $2.6 \pm 0.3$ & 1.40 & 2.16 \\
379.01 & 292 & $422 \pm 24$ & $1.6 \pm 0.1$ & $2.1 \pm 0.1$ & $3.1 \pm 0.2$ & 2.58 & 3.38 \\
379.02 & 136 & $196 \pm 11$ & $1.1 \pm 0.1$ & $1.40 \pm 0.04$ & $2.1 \pm 0.1$ & 1.83 & 2.31 \\
387.01 & 1122 & $1137 \pm 75$ & $3.3 \pm 0.3$ & $3.4 \pm 0.1$ & $29.4 \pm 73.4$ & 2.18 & 2.23 \\
401.01 & 2103 & $2363 \pm 132$ & $4.1 \pm 0.2$ & $4.9 \pm 0.1$ & $13.8 \pm 3.1$ & 6.57 & 7.82 \\
401.02 & 1618 & $1818 \pm 101$ & $4.2 \pm 0.2$ & $4.3 \pm 0.1$ & $12.1 \pm 2.7$ & 6.67 & 6.85 \\
433.01 & 2864 & $3048 \pm 76$ & $5.10 \pm 0.04$ & $5.5 \pm 0.1$ & $21.8 \pm 4.2$ & 5.60 & 6.06 \\
433.02 & 13690 & $14570 \pm 365$ & $11.7 \pm 0.1$ & $12.1 \pm 0.2$ & $47.6 \pm 9.3$ & 12.90 & 13.27 \\
592.01 & 539 & $561 \pm 19$ & $2.6 \pm 0.1$ & $2.37 \pm 0.04$ & $11.7 \pm 4.9$ & 2.74 & 2.48 \\
626.01 & 374 & $378 \pm 4$ & $1.8 \pm 0.1$ & $1.94 \pm 0.01$ & $19.2 \pm 8.7$ & 2.09 & 2.30 \\
628.01 & 476 & $504 \pm 21$ & $2.2 \pm 0.2$ & $2.24 \pm 0.05$ & $9.3 \pm 3.4$ & 1.87 & 1.90 \\
641.01 & 1002 & $2225 \pm 90$ & $3.1 \pm 1.3$ & $4.7 \pm 0.1$ & $4.3 \pm 0.1$ & 1.83 & 2.82 \\
644.01 & 23950 & $30367 \pm 591$ & $13.87 \pm 0.03$ & $17.4 \pm 0.2$ & $33.7 \pm 1.2$ & 33.16 & 41.67 \\
645.01 & 201 & $239 \pm 5$ & $1.61 \pm 0.05$ & $1.55 \pm 0.02$ & $3.6 \pm 0.2$ & 2.53 & 2.44 \\
645.02 & 257 & $305 \pm 7$ & $1.59 \pm 0.03$ & $1.75 \pm 0.02$ & $4.0 \pm 0.2$ & 2.51 & 2.75 \\
658.01 & 505 & $517 \pm 8$ & $2.1 \pm 0.1$ & $2.27 \pm 0.02$ & $14.7 \pm 5.0$ & 2.03 & 2.18 \\
658.02 & 484 & $496 \pm 8$ & $2.1 \pm 0.1$ & $2.23 \pm 0.02$ & $14.4 \pm 4.9$ & 2.02 & 2.13 \\
658.03 & 166 & $170 \pm 3$ & $1.2 \pm 0.1$ & $1.30 \pm 0.01$ & $8.5 \pm 2.9$ & 1.14 & 1.25 \\
703.01 & 130 & $131 \pm 1$ & $1.04 \pm 0.05$ & $1.142 \pm 0.003$ & $17.9 \pm 12.1$ & 1.36 & 1.50 \\
721.01 & 276 & $284 \pm 2$ & $1.63 \pm 0.03$ & $1.685 \pm 0.007$ & $9.9 \pm 1.4$ & 2.76 & 2.86 \\
841.01 & 2967 & $3071 \pm 45$ & $5.4 \pm 0.1$ & $5.54 \pm 0.04$ & $29.6 \pm 6.3$ & 5.44 & 5.56 \\
841.02 & 4962 & $5136 \pm 7$ & $7.0 \pm 0.2$ & $7.2 \pm 0.1$ & $38.3 \pm 8.1$ & 7.05 & 7.19 \\
1375.01 & 2608 & $2651 \pm 99$ & $5.3 \pm 0.6$ & $5.1 \pm 0.1$ & $40.0 \pm 44.8$ & 6.65 & 6.44 \\
\hline
\end{tabular}

Notes. In this calculation, we have taken into account only the closest objects $(<3$ arcsec) to the KOI. KOI-0704.01 is not present owing to the large errors in its $\Delta z$ value. ${ }^{(a)}$ New planet-to-star radii ratio assuming no limb-darkening. ${ }^{(b)}$ Planet-to-star radius assuming that the host is actually the secondary companion detected at less than 3 arcsec. ${ }^{(c)}$ Planet radii calculated by the Kepler team (Batalha et al. 2012). ${ }^{(d)}$ Planet radii assuming the new depth and no limb-darkening. No error is presented since no error in the stellar radii is given.

located well above the empirical ZAMS ( $\sim 3$ mag brighter $)$, indicating that it is probably a foreground M-dwarf. This hypothesis agrees with the distance calculated by the SED fitting approach, $d=76_{-10}^{+15}$, which is significantly closer than the A component.

\subsection{2. $\mathrm{KOI}-0433$}

The companion to KOI-0433 is too faint and red for clear conclusions to be extracted about its nature. Our analysis suggests that it is a $M 5_{-2}^{+1}$ star probably bound to the main KOI. However, the primary target is also too faint $\left(m_{i}>14.5\right)$ and the large errors in the distance do not allow us to set constraints on the projected separation of the system. Nevertheless, owing to the position of the secondary object in the Hertzsprung-Russell (HR) diagram of Fig. 8, as in KOI-0641C, there may be a third component very close to KOI-0433B that is unresolved in our images. Hence, we consider this object as a possible false positive, although more observations need to be done.

\subsection{3. $\mathrm{KOI}-0298$}

The projected distance estimated for the assumed primary target is $118 \pm 18 \mathrm{pc}$ according to Sect. 8 . The stellar companion to this KOI has a $i-z$ color typical of early $\mathrm{M}$ dwarfs. Its position in the $M_{i}$ vs. $i-z$ diagram is in good agreement with a simultaneous formation of both objects. Thus, a relative separation of $231 \pm$ $35 \mathrm{AU}$ is derived for the possible binary system. Moreover, since both objects have very similar magnitudes, it is unclear which star is actually hosting the transient object. There is also a third very faint object in the SDSSi image, which is not detected in the SDSSz image acquired a few hours later in the same night probably because it is a background blue object.

\subsection{4. $\mathrm{KOI}-0379$}

The projected distance of this object was estimated to be $244 \pm$ 76 pc. Its posible companion, which has a spectral type earlier than K5, would be located, if bounded, at $463 \pm 165 \mathrm{AU}$ from the primary target. However, since these estimations were based on photometric values and the corresponding night was not photometric, we caution that the true errors are probably larger than the ones provided here.

\subsubsection{KOI-0645}

KOI-0645 is a two-planet candidate system. The location of the secondary star in the HR diagram suggests that it is physically bound to the candidate host star. Its estimated distance of $328 \pm$ 82 pc corresponds to a relative separation of $910 \pm 228$ AU.

\subsubsection{KOI-0401}

This is another star presumably hosting two planets. The stellar companion has been estimated to be a $\mathrm{K} 9$ star at a projected distance of $590 \pm 190 \mathrm{AU}$ assuming that the distance of $302 \pm$ $98 \mathrm{pc}$ derived for the primary is correct.

\subsection{7. $\mathrm{KOI}-0623$}

The nature of the stellar companion at 5.54 arcsec from the KOI-0623 still remains unclear in this work. Both of the possible assumptions of luminosity classes are suitable. If it were a giant K2 background star, it should be at $790_{-350}^{+250} \mathrm{pc}$. If it were instead a dwarf it may be bound to the A component since they are very close to each other despite lying outside the margins of error. 


\subsection{8. $\mathrm{KOI}-0658$}

This is a very faint companion with an approximate spectral type of M3 that was detected close to this KOI. Owing to its faintness, large errors are present in the determination of its projected distance, which is $790 \pm 330$ AU based on a $420 \pm 180$ pc distance from the primary. Moreover, since $\Delta m_{z} \approx 4.06$, the planet properties of the two transient objects to the KOI are almost unaffected.

\subsection{9. $\mathrm{KOI}-0703$}

This is the faintest close companion detected in our sample. A less significant than 3- $\sigma$ detection was achieved for this companion so that the results for this object may be carefully used.

\subsubsection{0. $\mathrm{KOI}-1375$ and $\mathrm{KOI}-0387$}

A very close visual stellar companion was detected for these KOIs. In the case of KOI-1375, the object seems to be bluer than the KOI, suggesting that it is probably a background object. The companion of KOI-0387 has the same $i-z$ color, hence, if we assume that they have the same age we conclude that they also have similar spectral types. However, the KOI is several magnitudes brighter and redder than the companion, implying that it is most likely a background close companion. We also note that observations of KOI-0387 were performed during a non-photometric night. Very large errors were found owing to both the faintness and closeness of both objects to their corresponding KOIs.

\section{Conclusions}

We have started a high-resolution imaging follow-up of Kepler planet-host candidates. The main goal of this survey has been to provide additional confirmation of the planetary nature of these candidates and identify which are possible false positives. A total of 98 KOIs (out of the 997) from the second release of the Kepler team were observed using the lucky imaging technique with the AstraLux instrument at the $2.2 \mathrm{~m}$ telescope at Calar Alto Observatory.

Our main results show that the $58.2 \%$ of the KOIs are actually isolated in terms of not having any visual or bounded companion within 6 arcsec. In other words, $41.9 \%$ of the candidates have nearby objects. This is important for the following results: (a) false-positive rate determination, since it points directly to objects with stellar companions possibly mimicking a planet transit detected by Kepler, which are then the highest priority for a deeper follow-up with ground-based telescopes to determine the nature of this transit; (b) updating the planet properties, since as we have shown in Sect. 4, these depend on the brightness of the host star. We caution that the orbital and physical parameters of the 23 planets orbiting the 17 KOIs with close stellar companions should be revised; (c) estimating the binary rate in planet-host stars. According to their position on a $M_{i}$ vs. $i-z$ color-magnitude diagram, we have shown that between 6 and 9 of the close companions could actually be bound to the host star owing to their position in our empirical ZAMS. Their distances agree with those of an S-type binary, although more observations are necessary to confirm both the planet and the binary in all cases. The KOIs 0379B, 0658B, 0641B, 0645B, and $0298 \mathrm{~B}$ clearly lie in the ZAMS, which suggests that they simultaneously formed with their primary stars. Moreover, the
KOIs 0433B, 0401B, 0592B, and 0703B could also lie inside the error bars of the ZAMS although more work is needed to confirm this result. If confirmed, this would imply a lower limit to the observed binary frequency of $6.2-9.2 \%$. For the mediumdistance companions (3-6 arcsec), we found that only one of them (KOI-0623B) is possibly bound although this result may be unreliable owing to the large errors in the distance estimates.

Finally, we have provided accurate astrometric positions and $i$ magnitudes for the close and medium-distance companions, which could be used to re-compute planet-star parameters in the KOIs affected by the light of the companion. These results provide tighter constraints on theoretical studies of the false positive probabilities for the particular objects studied in this paper.

Acknowledgements. This research has been funded by Spanish grants AYA 2010-21161-C02-02, CDS2006-00070 and PRICIT-S2009/ESP-1496. J. Lillo-Box thanks the CSIC JAE-predoc program for the Ph.D. fellowship. H. Bouy is funded by the the Ramón y Cajal fellowship program number RYC-2009-04497. We appreciate the data-sharing and open discussions with the Kepler Science Team, specially Natalie Batalha and David Ciardi. After we had initiated this program in 2011 and contacted them, they were very cooperative with our efforts, with the general goal of maximizing the output of the Kepler mission, in a truly scientific and generous way. We also thank Calar Alto Observatory, both the open TAC and Spanish GTO panel for allocating our observing runs, and Felix Hormuth for providing such useful information about the AstraLux instrument.

\section{References}

Adams, E. R., Ciardi, D. R., Dupree, A. K., et al. 2012, AJ, 144, 42 Batalha, N. M., Rowe, J. F., Bryson, S. T., et al. 2012, ApJS, submitted [arXiv: 1202. 5852]

Bayo, A., Rodrigo, C., Barrado Y Navascués, D., et al. 2008, A\&A, 492, 277

Bertin, E., \& Arnouts, S. 1996, A\&AS, 117, 393

Borucki, W. J., Koch, D. G., Basri, G., et al. 2011, ApJ, 736, 19

Brandner, W., Zinnecker, H., Alcalá, J. M., et al. 2000, AJ, 120, 950

Cutri, R. M., Skrutskie, M. F., van Dyk, S., et al. 2003, VizieR Online Data Catalog, J/A\&A/2/146

Daemgen, S., Hormuth, F., Brandner, W., et al. 2009, A\&A, 498, 567

Desert, J.-M., Charbonneau, D., Fressin, F., Ballard, S., \& the Kepler Team 2011, Am. Astron. Soc., ESS meeting, 2, 403

Desidera, S., \& Barbieri, M. 2007, A\&A, 462, 345

Doyle, L. R., Carter, J. A., Fabrycky, D. C., et al. 2011, Science, 333, 1602

Duchêne, G. 1999, A\&A, 341, 547

Eggenberger, A., Udry, S., \& Mayor, M. 2004, A\&A, 417, 353

Holman, M. J., \& Wiegert, P. A. 1999, AJ, 117, 621

Hormuth, F. 2007, AstraLux diploma thesis (University of Heidelberg)

Howell, S. B., Everett, M. E., Sherry, W., Horch, E., \& Ciardi, D. R. 2011, AJ, 142,19

Jenkins, J. M., Caldwell, D. A., Chandrasekaran, H., et al. 2010, ApJ, 713, L87

Kley, W. 2010, in EAS Publ. Ser., 42, eds. K. Gożdziewski, A. Niedzielski, \& J. Schneider, 227

Law, N. M., Mackay, C. D., \& Baldwin, J. E. 2006, A\&A, 446, 739

Marcy, G. W., \& Butler, R. P. 1996, ApJ, 464, L147

Mayor, M., \& Queloz, D. 1995, Nature, 378, 355

Monet, D. G., Levine, S. E., Canzian, B., et al. 2003, AJ, 125, 984

Moraux, E., Bouvier, J., Stauffer, J. R., \& Cuillandre, J.-C. 2003, A\&A, 400, 891

Morton, T. D., \& Johnson, J. A. 2011, ApJ, 738, 170

Mugrauer, M., \& Neuhäuser, R. 2009, A\&A, 494, 373

O'Donovan, F. T., Charbonneau, D., Torres, G., et al. 2006, ApJ, 644, 1237

Ofek, E. O. 2008, PASP, 120, 1128

Pickles, A. J. 1998, VizieR Online Data Catalog, 611, 863

Raghavan, D., McAlister, H. A., Henry, T. J., et al. 2010, ApJS, 190, 1

Schmidt-Kaler, T. 1982, Physical parameters of the stars (New York:

Landolt-Börnstein New Series Springer-Verlag), 2B

Seager, S., \& Mallén-Ornelas, G. 2003, ApJ, 585, 1038

Smith, J. A., Tucker, D. L., Kent, S., et al. 2002, AJ, 123, 2121

Stello, D., \& Nissen, P. E. 2001, A\&A, 374, 105

Strehl, K. 1902, Astron. Nachr., 158, 89

Thebault, P. 2011, Celest. Mech. Dyn. Astron., 111, 29

Yanny, B., Guhathakurta, P., Bahcall, J. N., \& Schneider, D. P. 1994, AJ, 107, 1745 